$8{ }^{2}$ Department of Immunology and Microbial Disease, Albany Medical College, Albany, NY $9 \quad 12208$

\section{TCF-1 in CD8 T cells separates GVHD from GVL}

\author{
Rebecca Harris ${ }^{1}$, Mahinbanu Mammadli ${ }^{1}$, Adriana May ${ }^{1}$, Qi Yang ${ }^{2}$, Ivan Ting Hin Fung ${ }^{2}$, Jyoti \\ Misra Sen ${ }^{3,4}$, Mobin Karimi ${ }^{1 *}$
}

${ }^{3}$ National Institute on Aging-National Institutes of Health, 08C218,Biomedical Research Center, 251 Bayview Boulevard, Suite 100, Baltimore, MD 21224

${ }^{4}$ Immunology Program, Department of Medicine, Johns Hopkins School of Medicine, Baltimore, MD 21224.

To whom correspondence should be addressed:

Mobin Karimi

Assistant Professor of Immunology and Microbiology

SUNY Upstate Medical University,

766 Irving Ave Weiskotten Hall Suite 2281,

Syracuse, NY 13210

Office Phone: 315-464-2344

Laboratory Phone: 315-464-7652

Email: karimim@upstate.edu

Conflict of interest statement: The authors have declared that no conflict of interest exists 


\section{Abstract}

37 Allogeneic hematopoietic stem cell transplantation (allo-HSCT) is a curative treatment for

38 hematological malignancy due to graft-versus-leukemia (GVL) effects, mediated by alloreactive

39 donor T cells. However, these same donor cells cause graft-versus-host disease (GVHD), a life-

40 threatening complication. During GVHD, donor T cells proliferate, migrate, and produce

41 cytokines, leading to damage of healthy host tissues. Separating the linked processes of GVHD

42 and GVL is critical to improving outcomes. Here we show that TCF-1, a major T cell

43 developmental factor, plays a role in regulation of these processes. Using a mouse model of allo-

44 HSCT leading to GVHD, we found that conditional loss of TCF-1 in mature CD8 T cells

45 separates GVHD from GVL, providing an optimal clinical phenotype. This occurred due to

46 changes in cytokine production, proliferation, cell survival, chemokine receptor expression, and

47 gene expression. Thus, modulation of TCF-1 signaling reduces GVHD severity and persistence,

48 but maintains GVL effects, and could be useful for allo-HSCT or other T cell-mediated

49 disorders. Furthermore, here and in our companion manuscript we show that regulation of

50 mature, alloactivated T cells by TCF-1 differs between CD4 and CD8 T cells, suggesting that

51 TCF-1 is critical for regulation of mature alloactivated T cells, with unique programs in each

52 subset.

53

54

55

56

57

58

59

60

61

62

63 
64

65

66

67

68

69

70

71

72

73

74

75

76

77

78

79

80

81

82

83

84

85

86

87

\section{Introduction}

Allogeneic hematopoietic stem cell transplantation (allo-HSCT) is a curative therapy often used

for hematological malignancies (1). Mature donor T cells found in the graft become alloactivated upon recognition of host HLA as non-self $(2,3)$. This leads to T cell proliferation, migration to target organs, and cytokine production(4) These T cell activities help to clear residual malignant cells, which is called the graft-versus-leukemia effect (GVL)(5) (6). However, 30 to 70 percent of patients also experience graft-versus-host disease (GHVD), a serious complication driven by the same mature donor T cells (6). Damage to healthy host tissues during GVHD is a major problem for widespread use of allo-HSCT (7-9).Our work and that of others focuses on modulating donor T cell signaling to separate the linked processes of GHVD and GVL (9-11). In this manuscript, as well as in our companion manuscript, we address the role of T Cell Factor-1 (TCF-1), a major T cell developmental transcription factor, in regulating these processes(12)

TCF-1 is involved in the Wnt signaling pathway, and is critical for T cell development as well as activation (12-14) . However, it in unknown whether TCF-1 also regulates mature alloactivated T cells, and whether modulation of this factor may affect GVHD or GVL. TCF-1 has been reported to control Eomesodermin (Eomes) and possibly T-box transcription factor 21 (T-bet), two downstream transcription factors which are known to be involved in GVHD and GVL (10, 15, 16). Therefore, we hypothesized that TCF-1 may also affect and possibly separate these processes. all of the major T cell functions, as well as phenotypes, clinical outcomes, and gene expression, in a single model. We also used a unique mouse strain which has deletion of TCF-1 in mature T cells, rather than a global deletion (17). This Tcf7 flox/flox x CD4cre mouse experiences 
88 deletion of TCF-1 in all T cells at the double-positive phase of development, when all T cells

89 express CD4 (17). This allows us to overcome the severe T cell developmental defect that occurs

90 in global TCF-1 deletion, as TCF-1 is critical for the double-negative stage of development (17) .

91 Using these tools, we found that loss of TCF-1 reduces both the severity and the persistence of

92 CD8 T cell-driven GVHD, but maintains GVL functionality of mature alloactivated CD8 T cells.

93 For GVHD reduction, this is primarily due to reduced cytokine production, increased

94 predisposition to apoptosis, increased exhaustion, and possibly reduced proliferation of

95 alloactivated donor T cells, as well as increased central memory cells at baseline (18-20). GVL

96 effects are maintained against liquid tumors, due to increased donor T cell activation and

97 production of the cytotoxic mediators perforin and Granzyme B $(21,22)$. We showed that loss of

98 TCF-1 induces T cell-intrinsic phenotypic changes which promote GVL effects while disrupting

99 GVHD, including increased expression of Eomes, T-bet, CD122, CD44, and CD8 central

100 memory. The frequency of activating/transitioning CD8 T cells dropped when TCF-1 was lost.

101 Loss of TCF-1 affected expression of chemokine receptors on donor T cells, which was

102 previously unknown (18).

103

TCF-1 loss also affected pathways for T cell differentiation, cell adhesion, signaling

104 (MAPK, NFkB, calcium, TCR, NOD, TNF, and others), cell cycle, necroptosis, cell senescence, 105 autophagy, metabolism, and DNA replication (22).

106 Recent reports have identified a novel subset of CD8+ T cells which express CXCR5, a

107 marker traditionally associated with $\mathrm{T}$ follicular helper cells (23). These CXCR5+ CD8+ T cells

108 are thought to be stem-like, and help to maintain pools of CD8 T cells during chronic responses

109 to antigen (24). These cells have been described in situations where antigen is persistently

110 present, such as chronic infections (25). We hypothesized that these cells may also play a role in 
111 GVHD, where damaging disease persists over time, and exposure to alloantigen in the host is

112 chronic (26). Indeed, we found that CXCR5+ CD8+ T cells are present in mice experiencing

113 GVHD from WT CD8+ T cells. The peak frequency of these cells correlated temporally with

114 peak clinical score. Additionally, mice given TCF cKO CD8+ T cells had lower frequencies of

115 donor-derived CXCR5+ CD8+ T cells, suggesting that these cells may be important for

116 sustaining GVHD responses over time.

117 Therefore, TCF-1 controls mature CD8 T cell phenotype and functions during

118 alloactivation, a novel finding. Together with our companion paper, we have shown that

119 regulation of mature alloactivated CD4 versus CD8 T cells by TCF-1 is different. Modulation of

120 TCF-1 and Wnt signaling may be a successful option for separating GVHD from GVL to

121 improve patient outcomes following allo-HSCT. We also identified a role for CXCR5+ CD8+ T

122 cells in GVHD, providing another potential target for T cell modulation to improve patient

123 outcomes.

124

125

126

127

128

129

130

131

132

133

134

135

136

137

138

139

140

141

142 


\section{Results}

144

\section{Loss of TCF-1 in donor CD8 T cells reduces severity and persistence of GVHD symptoms.}

146 Most of the previous research on TCF-1 utilized a global TCF-1 knockout, because the primary

147 focus was on TCF-1's role as a developmental factor. However, we wanted to study the role of

148 TCF-1 in mature T cells. Global loss of TCF-1 results in minimal T cell production from the

149 thymus, because TCF-1 is critical for DN stages of development. Therefore, we obtained mice

150 with a T cell-specific knockout for TCF-1 (Tcf7 flox/flox x CD4cre, called TCF cKO here (17).

151 This allowed us to study mature T cells that developed normally in the thymus, then lost 152 expression of TCF-1 at the DP phase.

To determine whether TCF-1 plays a role in mature alloactivated T cell regulation, which

is currently unknown, we used a mouse model of MHC-mismatched allo-HSCT leading to

GVHD and GVL. Briefly, BALB/c mice (MHC haplotype d) were lethally irradiated and

transplanted with BALB/c bone marrow and C57B1/6-background (MHC haplotype b) donor

CD8 T cells (10). The donor cells came from wild-type (WT), CD4cre-positive (CD4cre), or

TCF-1-deficient (TCF cKO) mice. Recipients were given $1 \times 10^{6}$ CD8 T cells and $10 \times 10^{6}$

BALB/c T cell-depleted bone marrow cells, as well as $2 \mathrm{X} 10^{5} \mathrm{~B}$-cell acute lymphoblastic

BALB/c mice and allogeneic to C57BL/6 (B6) mice (10). The MHC haplotype mismatch

165 The mice were scored based on six factors: skin integrity, fur texture, posture, activity level, 

severity, with a high peak score around day 7, suggesting severe GVHD (Fig. 1A). This high score was maintained until the recipient mice died of disease, suggesting persistent disease (Fig. 1A). Disease burden, as determined by the percent of mice in each group that died during the experiment, was high for WT-transplanted mice (Fig. 1B). WT-transplanted mice lost weight

172 initially, and were unable to regain much weight (Supp. Fig. 1A). Most mice that received WT T

173 cells also died prior to day 60 (Fig. 1A-C). Recipient mice given CD4cre T cells showed no

174 significant differences in survival or clinical score from those given WT T cells (Fig. 1A-C),

175 (Supp. Fig. 1A). In contrast, mice given TCF cKO T cells had much better survival, lower peak

176 and average clinical scores, lower disease burden, and a gain in weight following the initial

177 weight loss period (Fig.1A-C), (Supp. Fig. 1A). In addition, the clinical scores for these mice

178 quickly reduced to near-control levels following peak score at day 7 (Fig. 1A), suggesting that

179 disease does not persist in these mice. Therefore, loss of TCF-1 in donor T cells led to reduced

180 GVHD severity and persistence, with improved survival. pieces of skin, small intestine, and liver from recipient BALB/c mice. These organs were fixed, sectioned, stained with hematoxylin and eosin (H\&E), and scored by a pathologist (A.M.) blinded to study group and donor type (Fig. 1D). At day 7, there is little to no difference observed between WT- and TCF cKO-transplanted organs, except for a trend toward reduced median grade in the gut for TCF cKO-transplanted mice (Supp. Fig. 1B). This correlates with 
190 the observation that until day 7, disease course appears similar in both groups, with a decreased

191 peak score appearing at day 7 (Fig. 1A). By day 21, significant differences in the number of mice

192 per group with severe versus mild GVHD grades (3-4 for severe versus 0-2 for none to

193 moderate)(29)(30) for the liver and skin were observed, with WT-transplanted mice having more

194 severe disease (Fig. 1E). In addition, the median grade for all organs trended towards decreased

195 for TCF cKO-transplanted mice, which correlated with the lymphocyte infiltration and tissue

196 damage seen in slide photos (Supp. Fig. 1B-C). These changes were already visible by day 14 as

197 a trend, suggesting that TCF cKO-transplanted mice have altered disease course after peak score

198 at day 7 (Supp. Fig. 1B-C). Altogether, these data suggest that TCF-1 normally promotes

199 GVHD damage to healthy tissues, and is indispensable for T cells to cause this damage. Thus,

200 loss of TCF-1 in donor T cells leads to reduced severity and persistence of GVHD over time.

201

202 TCF-1-deficient CD8 T cells retain anti-tumor capabilities for the graft-versus-leukemia

203 (GVL) effect. We showed that TCF-1-deficient donor T cells reduce the severity and persistence

204 and GVHD. However, it is clinically optimal if GVL effects are maintained despite reduced

205 GVHD, because the same donor T cells mediate both effects. Therefore, we tested whether GVL

206 effects were compromised by loss of TCF-1 in donor T cells. We used the allo-HSCT mouse

207 model described in Fig. 1 to assess GVL responses. The B-ALL cells expressed luciferase (10),

208 allowing us to track them by injecting D-luciferin into the recipient mice, and imaging them with

209 an in vivo bioluminescence scanner (IVIS 200). The mice were weighed, scored, and scanned

210 three times a week until the end of the experiment (Fig. 2).

211 At day 1 post-transplant, recipient mice from all groups (B-ALL luc+BM only, B-ALL

212 luc+BM+WT CD8 T cells, B-ALL luc +BM+TCF cKO CD8 T cells, and B-ALL luc 
$+\mathrm{BM}+\mathrm{CD} 4 \mathrm{cre}$ CD8 T cells) showed detectable levels of tumor cells in their bodies (Fig. 2A).

$2142 \times 10^{5}$ tumor cells were injected, so they were immediately detectable, but not overwhelming for

215 the donor T cells(10). Over time, the group receiving only bone marrow and tumor cells showed

216 a large increase in tumor growth (Fig. 2A-B), because no T cells were present to control the

217 tumor cells. In contrast, most mice given CD8 T cells from any donor type along with the BM

218 and B-ALL luc cells were able to clear the tumor cells by the end of the experiment (Fig. 2A-B).

219 The GVL effect was maintained even in CD4cre and TCF cKO-transplanted mice, with little

220 difference in quantified bioluminescence between groups (Fig. 2B). Tumor burden was also

221 similar between WT and TCF cKO-transplanted mice (Fig. 2C). As seen before in Fig 1, mice in

222 the WT and CD4cre groups died from GVHD over time, but mice in the TCF cKO group had

223 mild GVHD that resolved over time (Fig. 1A,2A). Altogether, these data show that TCF-1 is

224 dispensable for GVL effects, but critical for GVHD. Therefore, loss of TCF-1 in donor T cells

225 provides a clinically optimal phenotype, where GVHD severity is reduced but beneficial GVL

226 effects are maintained.

Loss of TCF-1 drives changes to mature CD8 T cell phenotype which are primarily cell-

229 intrinsic. Alloactivated T cells must survive, proliferate, produce cytokines, and migrate to target

230 organs in order to mediate GVHD or GVL. The phenotype of the donor T cell can also affect its

231 ability to perform these functions and contribute to GVHD/GVL. To determine whether loss of

232 TCF-1 affected mature donor T cell phenotype, we performed flow cytometry phenotyping on

233 pre-transplant donor CD8 T cells (Fig. 3A-E). To begin, we looked at expression of

234 Eomesodermin (Eomes) and T-box transcription factor 21 (T-bet), both of which are downstream

235 of TCF-1. Eomes and T-bet both are known to contribute to GVHD, and Eomes is also critical 
236 for GVL effects $(10,31)$. Some reports have claimed that Eomes is activated by TCF-1 (meaning

237 loss of TCF-1 reduces Eomes expression (15). However, in our model of conditional TCF-1

238 deletion, we found that TCF cKO CD8 T cells have increased expression of Eomes compared to

239 WT CD8 T cells (Fig. 3A). Other reports have claimed that T-bet may be activated or not

240 affected by TCF-1 (14), but we found that loss of TCF-1 led to increased T-bet expression in

241 CD8 T cells (Fig. 3B). This suggests that TCF-1 normally suppresses expression of Eomes and

242 T-bet in mature T cells. Next, we looked at T cell activation using CD44 and CD122Expression

243 of CD122 was increased in TCF-1-deficient donor cells (Fig. 3C). CD44 expression was

244 polarized more potently among CD8 T cells from TCF cKO mice (Fig. 3D-E). Some reports

245 have suggested that CD44 ${ }^{\text {hi }}$ T cells do not cause GVHD or cause less severe GVHD $(10,32)$.

246 Therefore, using CD62L and CD44, we identified four major memory subsets: central memory

247 (44+62L+), effector memory (44+62L-), naive (44-), and activating/transitioning (44 mid) cells.

248 TCF cKO mice had increased central memory CD8 T cells, and decreased

249 transitioning/activating CD8 T cells (Fig. 3E). Thus, loss of TCF-1 results in a more memory-

250 skewed phenotype for CD8 T cells. Some reports have suggested that memory T cells delay

251 induction of $\operatorname{GVHD}(10,32)$, so this phenotypic change may be beneficial.

252 Changes to cell phenotype in a knock-out mouse may be cell-intrinsic (due directly to

253 gene deficiency within the cell) or cell-extrinsic (due to changes in the microenvironment from

254 gene deficiency). To determine whether the phenotypic effects we observed were cell-intrinsic or

255 cell-extrinsic, we developed a chimeric mouse model. Briefly, we mixed bone marrow from WT

256 and TCF cKO mice at a 1:4 (WT:TCF) ratio for a total of 50x10 6 BM cells, then used this

257 mixture to reconstitute lethally irradiated Thy1.1 mice. We used a 1:4 ratio to ensure survival of

258 the KO cells (based on our initial observations that TCF cKO T cells do not proliferate well in 
culture). At 9 weeks post-transplant, blood was taken to ensure reconstitution and survival of

260 both donor types in each mouse. At 10 weeks, splenocytes were phenotyped by flow cytometry,

261 with donor cells being identified by H2Kb, CD45.1 (WT), and CD45.2 (TCF cKO) markers (Fig.

262 3F-I). TCF cKO donor CD8 T cells from the chimera still had increased expression of T-bet

263 (Fig. 3G), and expression of Eomes and CD122 were also increased to near-KO levels in WT

264 donor cells (Fig. 3F,H). This suggests that while phenotypic changes are primarily cell-intrinsic

265 in the TCF cKO mice, some cell-extrinsic effects are also present. CD8 central memory cells

266 were decreased in TCF cKO cells from the chimera (rather than being increased as in pre-

267 transplant cells), suggesting that this effect is cell-extrinsic (Fig. 3I). Chimeric TCF cKO donor

268 cells also showed an increase in naive cells, which was unique to the chimera (Fig. 3I). The

269 decrease in transitioning cells was also preserved in chimeric TCF cKO cells, suggesting it is a

270 cell-intrinsic effect (Fig. 3I). Thus, the phenotypic changes induced in CD8 T cells by loss of

271 TCF-1 are primarily cell-intrinsic, with some additional cell-extrinsic effects.

272

273 Loss of TCF-1 alters cytokine and cytotoxic mediator production by mature alloactivated CD8

$274 T$ cells. One of the hallmarks of GVHD is the release of pro-inflammatory cytokines and

275 cytotoxic mediators by alloactivated donor T cells, eventually leading to cytokine storm. These

276 mediators also drive the GVL effect, which kills residual malignant cells. We examined whether

277 loss of TCF-1 in donor CD8 T cells led to changes in cytokine or cytotoxic mediator production,

278 thereby affecting GVHD damage. We allotransplanted lethally irradiated BALB/c mice as

279 described above, with mice receiving $1.5 \times 10^{6} \mathrm{WT}$ or TCF cKO CD3 donor T cells, along with T

280 cell-depleted BALB/c bone marrow. Recipients were sacrificed at day 7 post-transplant.

281 Splenocytes were isolated and restimulated by 6 hours of culture with PBS (control) or anti- 
282 CD3/anti-CD28 (stimulation), along with Golgiplug. Afterwards, the cultured cells were stained 283 with antibodies against $\mathrm{H} 2 \mathrm{~Kb}, \mathrm{CD} 3, \mathrm{CD} 4, \mathrm{CD} 8$, TNF- $\alpha$, and IFN- $\gamma$. Our data showed that 284 production of TNF- $\alpha$ by donor CD8 T cells trended toward decreasing when TCF-1 was lost 285 (Fig. 4A). In contrast, IFN- $\gamma$ trended toward increasing upon loss of TCF-1 in CD8 T cells (Fig. 286 4B). This suggests that TNF- $\alpha$ is more critical for GVHD-related damage by $\mathrm{T}$ cells than IFN- $\gamma$ 287 is. We also obtained serum from cardiac blood of recipient mice at day 7 post-transplant, and tested it with a mouse Th cytokine ELISA panel (LEGENDplex kit from Biolegend). TNF- $\alpha$ 290 in serum of mice given TCF cKO CD8 T cells was lower than in mice given WT CD8 T cells at day 7 (Fig. 4C). Additionally, TNF- $\alpha$ levels in serum appeared to decrease over time past day 7 for both groups of mice, especially those given WT T cells (Fig. 4C). When compared alone, serum from day 14 also showed a significant drop in TNF- $\alpha$ levels for TCF cKO-transplanted mice (Fig. 4D). Interestingly, the same trend of decreasing levels in TCF cKO-transplanted mice was seen for IFN- $\gamma$ in the serum (Fig. 4E-F). This suggests that TCF cKO CD8 T cells may be capable of more IFN- $\gamma$ production than WT cells when restimulated, but in reality, produce less IFN- $\gamma$ than WT cells when allotransplanted. We also examined IL-2 levels, and found that they were increased in TCF cKO transplanted mice at day 7, supporting the early rise in proliferation, but drop below WT levels at day 14 and 21, supporting the hypothesized drop in proliferation as disease resolves (Fig. 4G). mediate GVL effects (33). We looked at perforin and Granzyme B protein levels by performing a 303 western blot on lysates from naive CD8 T cells or allotransplanted CD8 T cells from WT and 304 TCF cKO mice. We found that naive TCF cKO CD8 T cells show a trend towards producing 
more perforin and Granzyme B than WT CD8 T cells (Fig. 4H-J). We also found that

306

307

308

309

310

311

312

313

314

315

316

317

318

319

320

321

322

323

324

325

326

327

alloactivated TCF cKO CD8 T cells trend towards still producing more Granzyme B than WT

CD8 T cells, with little to no difference in perforin expression compared to WT (Fig. 4H,K-L).

These results suggest that the GVL effect is maintained by TCF cKO T cells because they have more expression on a per-cell basis of cytotoxic mediators which are critical to this process.

\section{TCF-1 controls chemokine and chemokine receptor expression of donor CD8 T cells during}

alloactivation. One of the major functions of alloactivated T cells is migration from spleen to GVHD target organs, including liver and small intestine. Given that GVHD severity is significantly reduced when donor T cells lack TCF-1, we examined whether these donor cells have defects in migration to target organs. To do this, we allotransplanted lethally irradiated $\mathrm{BALB} / \mathrm{c}$ mice as described above, but with the donor cells being a 1:1 mixture of WT:TCF cKO (or WT:WT as control) CD3+ T cells. A total of $1 \times 10^{6} \mathrm{~T}$ cells were injected, and prior to transplant the donor cells were checked to ensure a 1:1 ratio of donor types, as well as to ensure an approximately 1:1 distribution of CD4 and CD8 T cells (Fig. 5A). WT Ly5.1 donors were identified with CD45.1, and TCF cKO or WT C57 donors were identified with CD45.2. At day 7, the spleen, lymph nodes, small intestine, and liver were removed from recipient mice and examined for expression of $\mathrm{H} 2 \mathrm{~Kb}, \mathrm{CD} 45.1$, and $\mathrm{CD} 45.2$ to determine relative migration of donor T cells (Fig. 5A).

For CD8 T cells, TCF cKO cells were present at slightly lower frequencies in guts, indicating a potential mild migration defect (Fig. 5A), (Supp. Fig. 2). However, this mild reduction in TCF cKO donor cells was also observed in the spleen, indicating that either a very mild migration defect, or more likely a mild proliferation defect, caused the decrease (Fig. 5A), 
328 (Supp. Fig. 2). Therefore, loss of TCF-1 likely does not significantly impact migration of 329 allaoctivated CD8 T cells to target organs.

330 Expression of chemokines and chemokine receptors is a critical aspect of T cell migration 331 to target organs. To determine whether expression of these molecules was affected by loss of 332 TCF-1 in CD8 T cells, we FACS sorted pre- and post-transplanted donor CD8 T cells from WT 333 or TCF cKO mice (spleen only for pre-transplant, spleen or liver for post-transplant). We then 334 extracted RNA from the cells, converted it to cDNA, and performed qPCR using a 96-well 335 mouse chemokine/chemokine receptor plate (Thermo Fisher). As expected, expression of these 336 markers was generally upregulated in alloactivated cells. Expression of these markers was higher 337 in TCF cKO CD8 T cells from pre-transplant spleen compared to WT pre-transplant spleen (Fig. 338 5B), but these markers were downregulated in TCF cKO CD8 T cells from post-transplant liver 339 and spleen compared to WT cells (Fig. 5C-D). Therefore, TCF-1 controls expression of CD8 T

340 cell chemokine/chemokine receptors, but loss of this factor has little to no impact on migration of 341 these cells to target organs. Of note, a migration defect may become more apparent as the disease 342 course continues, given that GVHD resolves over time in TCF cKO transplanted mice.

345 In order for GVHD to persist, donor T cells must proliferate in secondary lymphoid organs and 346 target organs $(34,35)$. Naive and effector T cells drive GVHD, but they are short-lived and must 347 be replaced to maintain an alloresponse $(35,36)$. Given that memory cells are increased and 348 transitioning/activating cells are decreased among CD8 T cells when TCF-1 is lost, we 349 hypothesized that proliferation, survival, or exhaustion of these cells may also be affected. To 350 examine proliferation of these cells, BALB/c mice were allotransplanted as described above, 
351 with mice receiving $1 \times 10^{6}$ donor CD3 T cells (WT or TCF cKO) and $10 \mathrm{X} 10^{6} \mathrm{BALB} / \mathrm{c} \mathrm{BM}$ cells.

352 Recipient mice were given $25 \mathrm{mg} / \mathrm{kg}$ of EdU in PBS (i.p. injection) on day 5 and 6 to label

353 proliferating cells. On day 7 post-transplant, we euthanized recipient mice and obtained

354 lymphocytes from spleen and liver. These cells were stained with an EdU click chemistry kit

355 (Invitrogen) to detect EdU-stained (proliferating) cells by flow cytometry. There was an increase

356 in proliferation in the spleen and liver by CD8 TCF cKO T cells, which was significant in the

357 liver (Fig. 6A-B). Therefore, proliferation at day 7 is increased in TCF cKO CD8 T cells. We

358 also used Ki67 staining in a similar but separate allo-HSCT experiment (without injecting EdU)

359 to confirm these results, as Ki67 is a marker of T cell activation and proliferation. Indeed, Ki67

360 expression was increased or trending towards increased for TCF cKO CD8 T cells in the spleen

361 and liver, respectively (Fig. 6C-D). Therefore, proliferation in CD8 T cells early after

362 alloactivation is increased by loss of TCF-1. However, given that symptoms of GVHD resolve

363 after day 7 in TCF cKO-transplanted recipients (Fig. 1A), we hypothesize that proliferation of

364 donor cells decreases below WT levels after this point.

365 Impaired survival of donor T cells following allotransplant could also explain the

366 reduction in disease. To test this, we performed a death assay by allotransplanting recipient mice

367 as described with $10 \mathrm{X} 10^{6} \mathrm{BALB} / \mathrm{c} \mathrm{BM}$ cells and $1 \mathrm{X} 10^{6}$ donor CD3 T cells (WT or TCF cKO).

368 At day 7 post-transplant, recipient mice were euthanized, we obtained cells from spleen and

369 liver, and stained them with antibodies against $\mathrm{H} 2 \mathrm{~Kb}, \mathrm{CD} 3, \mathrm{CD} 4$, and CD8, as well as Annexin

370 V-FITC, and LIVE/DEAD Near-IR. This allowed us to identify donor CD8 T cells, and further

371 to separate three distinct populations - live cells, dead cells, and apoptotic cells (Fig. 6E-F).

372 When we compared WT and TCF cKO donor cells in the spleen, we found that TCF cKO CD8 T

373 cells were trending towards less frequently dead, and more frequently live or apoptotic, than WT 
374 donor cells (Fig. 6E). There appeared to be no difference in apoptosis or cell death in the liver

375 (Fig. 6F). This suggests that CD8 T cells lacking TCF-1 in the spleen may be more prone to

376 apoptosis following allotransplant, suggesting a similar role for TCF-1 in survival of mature cells

377 as for developing cells in the thymus $(37,38)$. In support of this, when we attempted to sort CD8

378 T cells from WT and TCF cKO-transplanted mice at day 7 post-transplant (in a separate

379 experiment), there were significantly fewer CD8 donor T cells in the spleen from TCF cKO

380 donors than from WT donors (by number of cells/10,000 total sorted cells) (Supp. Fig. 3A).

381 Donor cells also trended towards reduced for TCF cKO cells in the liver at day 7 (Supp. Fig.

382 3B). In addition, we attempted to sort TCF cKO donor CD8 cells from recipient mice at day 14

383 post-transplant, and donor cells in the spleen and liver were trending towards reduced compared

384 to WT and TCF cKO donor CD8 T cells from day 7 (Supp. Fig. 3A-B). When we attempted to

385 culture TCF cKO T cells using anti-CD3/anti-CD28 with or without IL-2, we found that they

386 proliferate poorly in culture compared to WT T cells even when activated, making in vitro work

387 very difficult (empirical observations). Therefore, it is likely that survival and/or proliferation are

388 reduced after day 7 in TCF cKO CD8 T cells, giving rise to the progressive resolution in

389 symptoms observed in TCF cKO-transplanted mice.

390 Finally, it is also possible that the donor T cells lacking TCF-1 become exhausted shortly

391 after being transplanted. To determine whether this is occurring, we allotransplanted recipient

392 mice as described with $10 \times 10^{6} \mathrm{BALB} / \mathrm{c} \mathrm{BM}$ cells and $1 \times 10^{6}$ donor CD3 T cells (WT or TCF

$393 \mathrm{cKO}$ ). At day 7 post-transplant we stained cells from the spleen and liver for TOX, a marker of T

394 cell exhaustion. We found that donor CD8 T cells from TCF cKO mice were significantly more

395 exhausted in the spleen, and trending towards more exhausted in the liver, than WT donor cells

396 (Fig. 6G-H). Together, these data suggest that CD8 donor T cells from TCF cKO mice have 
more frequent apoptosis, increased exhaustion, and a potential progressive defect in proliferation or survival over time compared to WT CD8 T cells. This provides a clinically optimal phenotype, because the donor cells eradicate residual malignant cells quickly after being transplanted, but then die off or become non-functional, removing the threat of host tissue damage. This observation helps to explain why GVHD score reduces rather than persisting over

402 time for TCF cKO-transplanted mice (Fig. 1A), as donor TCF cKO T cells may no longer be 403 functional after day 7.

404

405

Loss of TCF-1 alters suppressive profile of T cells. To determine whether loss of TCF-1 also

406 impacted the suppressive profile of donor T cells, 1 X10 ${ }^{6} \mathrm{WT}$ or TCF cKO CD8 T cells were

407

408

409

410

411

412

413

414

415 allotransplanted into recipient BALB/c mice with $10 \times 10^{6} \mathrm{BALB} / \mathrm{c}$ BM cells as described. Lymphocytes were isolated from spleen and liver of the recipient mice at day 7 , day 14 , and day 21 post-transplant for phenotyping. We examined expression of PD-1, PD-L1, and CTLA-4

(Supp. Fig. 4). TCF cKO CD8 T cells had lower expression of PD-1 in both spleen and liver at day 14 and 21 post-transplant, but not day 7 post-transplant (Supp. Fig. 4-A-B). These data suggest that CD8 T cells from TCF cKO mice may be less susceptible to suppression by tumor cells (or other cells) expressing PD-L1 (39-41). Therefore, these T cells are more likely to be effective at killing target cells, because they are less capable of being turned off when a response is appropriate. However, this phenotypic change also increases the risk of autoreactive damage. Despite this, GVHD score and histology sections show reduced tissue damage for TCF cKOtransplanted mice (Fig. 1A-E). 
420

421

422

423

424

425

426

427

428

429

430

431

432

433

434

435

436

437

438

439

440

441

442

expressed on T cells is unknown, but it is thought to promote tumor tolerance by suppressing activation and promoting apoptosis of other T cells (42). Therefore, the higher expression of PDL1 suggests that $\mathrm{T}$ cells that do not migrate to peripheral tissues by later time points after transplant are capable of suppressing other T cells around them. Thus, the loss of TCF-1 may induce a $\mathrm{T}$ cell phenotype which is both less susceptible to external suppression and more capable of suppressing other T cells. This may result in enhanced tumor killing with minimal damage to healthy bystander cells.

Expression of CTLA-4 was unaffected by loss of TCF-1 in donor CD8 T cells, both in liver and spleen (Supp. Fig. 4E-F). CTLA-4 is responsible for turning off activation signaling resulting from TCR activation (43). Therefore, this data suggests that TCF cKO cells do not suffer from abnormal suppression or prolongation of TCR signaling due to improper regulation. These cells most likely are not always "on" or always "off" with regard to activation.

\section{Reduced CXCR5 expression in CD8 T cells of TCF-1 deficient mice may contribute to reduced}

GVHD severity. CXCR5 is a major T cell chemokine receptor which contributes to T cell

migration out of the spleen, although CXCR5 is traditionally described as a $\mathrm{T}$ follicular helper (Tfh) cell subset marker. In situations where antigen persists over a long period of time, such as during immunization or chronic viral infection, some CD8+ T cells also express CXCR5. These cells have been described as stem-like, and maintain the pool of exhausted CD8+ T cells during the immune response in these contexts (23). These cells are also responsible for proliferation and response to PD-1 blockade (44). In GVHD, alloantigens that are recognized by donor T cells come from the patient's HLA molecules, so these antigens are chronically present. We hypothesized that persistence of GVHD damage over time results from the presence of CXCR5+ 
$443 \mathrm{CD} 8+\mathrm{T}$ cells, which maintain the pool of cells capable of responding to alloantigen and causing 444 damage. In this model, CXCR5+ CD8+ T cells in the spleen proliferate continuously, allowing 445 waves of CD8 T cells to migrate to target organs, causing damage continuously.

446 To examine whether this hypothesis was correct, we looked at CXCR5 expression in 447 naïve and alloactivated WT CD8+ T cells (Fig. 7). In naïve WT T cells, CXCR5 expression 448 among CD8+ T cells was low (Fig. 7A). After alloactivation for 7, 14, or 21 days in 449 allotransplanted mice, donor splenic WT CD8+ T cells expressed CXCR5 at a higher frequency 450 than naïve cells(Fig. 7A-D). Importantly, this CXCR5 expression remained higher than naïve 451 levels at all timepoints after alloactivation, with a peak in expression around day 7 (Fig. 7A-D).

452 In addition, donor CD8+ T cells found in the liver also expressed CXCR5 at low levels, and this 453 expression did not change over time post-alloactivation (Fig. 7E-G). Together, these data 454 support the hypothesis that in allotransplanted mice receiving WT donor T cells, CXCR5+ CD8+ 455 T cells do exist. These cells persist over time, and may contribute both to ongoing damage, and 456 to continual migration of donor cells into the liver and other target organs. responsible for development of these Tfh-like CXCR5+ CD8+ T cells (45). Therefore, we examined whether CXCR5 expression is reduced when TCF-1 is lost in these cells. The

460 frequency of CXCR5+ CD8+ T cells is not significantly lower in naive TCF cKO mice than in 461 naive WT mice (Fig. 7A). However, upon alloactivation, CD8+ T cells from TCF cKO donor 462 mice had lower expression of CXCR5 in spleen at all time points, especially at day 14 and day 46321 (Fig. 7A-D). Although not significantly different from WT mice, the expression of CXCR5 464 among CD8+ donor $\mathrm{T}$ cells from the TCF cKO mice also trended towards decreasing over time 465 in the liver (Fig. 7E-G), suggesting that these cells do not persist or are not replaced by 
466 subsequent waves of migrating cells when TCF-deficient donor cells are used for transplant.

467 Thus, these data support the conclusion that TCF-1 is indeed important for development of

468 CXCR5+ CD8+ T cells, but loss of TCF-1 only in T cells does not completely abrogate their

469 development. Instead, frequencies of these cells are reduced in the TCF cKO donor cells during

470 allotransplant, which we hypothesize may lead to impaired migration, and thus, reduced

471 persistence and severity of GVHD-driven damage.

472

473 TCF-1 contributes to control of cell survival and immune signaling pathways in CD8 donor T

474 cells. Given that the phenotype and functions of donor T cells, as well as disease outcomes, were

475 significantly altered by loss of TCF-1 on donor cells, we sought to determine what specific gene

476 changes occurred to support this. We allotransplanted recipient BALB/c mice with $10 \times 10^{6}$

$477 \mathrm{BALB} / \mathrm{c} \mathrm{BM}$ and $1 \mathrm{X} 10^{6}$ donor CD3 T cells as above, and FACS-sorted pre- and post-transplant

478 WT or TCF cKO donor CD8 T cells, which were stored in Trizol . RNA sequencing was then

479 performed (prep by SUNY Upstate Molecular Analysis Core, sequencing by University at

480 Buffalo Genomics Core). Data were analyzed in Partek Flow (Fig. 8). We found that WT and

481 TCF cKO donor CD8 T cells cluster together both by strain and by timepoint (pre- and post-

482 transplant) (Fig. 8A). (Supp. Fig. 5A-C). Numerous genes were affected by loss of TCF-1 in

483 post-transplant donor cells, with cells in both spleen and liver affected (Fig. 8B). Changes to

484 Tbx21 (T-bet), as well as Cxcr4 and Ccr4 (chemokine receptors) were observed in pre-transplant

485 CD8 donor T cells lacking TCF-1 (Supp. Fig. 5D). As before, significant genes were determined

486 by $p$-value $\leq 0.05$ for pre-transplant cells. In post-transplant spleen, changes to IFNg (IFN- $\gamma$ ),

487 Tbx21 (T-bet), Prf1 (perforin), and Il2rb (CD122) were observed for TCF cKO cD8 T cells (Fig.

488 8C). In liver, TCF-1-deficient CD8 donor T cells showed changes to IFN- $\gamma$ and Cxcr6 
489 (chemokine receptor) (Fig. 8D). These data support our earlier findings that expression of

490 chemokines/chemokine receptors, as well as cytokines and cytotoxic mediators, are regulated by

491 TCF-1 in mature alloactivated CD8 T cells.

492 Pathway enrichment analysis in pre-transplant CD8 donor cells showed that loss of TCF-1

493 affected T cell differentiation, cell adhesion, many signaling pathways (MAPK, NFkB, calcium,

494 and others), and TCR signaling (Supp. Fig. 5E). This analysis was determined using p-values

495 rather than False Discovery Rate (FDR), as no significantly different features were detected with

496 FDR $\leq 0.05$. Following transplant, many pathways were affected by loss of TCF-1, in both spleen

497 and liver-derived donor CD8 T cells. In both organs, loss of TCF-1 impacted cell cycle,

498 necroptosis, cell senescence, and autophagy pathways (Fig. 8E-F). This supports the idea that

499 CD8 T cells are more prone to apoptosis or other forms of cell death when TCF-1 is lost, leading

500 to donor cell loss after allotransplant. In the liver, NOD- and TCR-signaling were also affected,

501 while in spleen, metabolism, DNA replication, T cell differentiation, NFKB signaling, and TNF

502 signaling were impacted (Fig. 8E-F).

503 Finally, we looked at expression levels of CXCR5 in D7 post-transplant donor CD8 T cells from

504 spleen and liver, comparing WT and TCF cKO samples. We found that CXCR5 expression level

505 was trending towards decreased in TCF cKO donor cells at D7 (Fig. 8G), confirming the results

506 that we obtained by flow cytometry (Fig. 7). Although CXCR5 was not identified as a

507 significantly changed gene (d7 spleen had p-value 0.041 but FDR 0.114, d7 liver had p-value

5080.074 and FDR 0.179), the fold change for this gene was relatively large in spleen (8.392), which

509 agrees with our flow cytometry data. Therefore, it is likely that loss of CXCR5 in CD8 T cells is

510 an important factor in the reduced GVHD seen from TCF-deficient donor cells. Overall, these

511 results show that TCF-1 controls a wide range of immune-related gene programs in mature and 
bioRxiv preprint doi: https://doi.org/10.1101/2021.03.22.436494; this version posted March 23, 2021. The copyright holder for this preprint (which was not certified by peer review) is the author/funder. All rights reserved. No reuse allowed without permission.

512 allaoctivated CD8 T cells. Loss of TCF-1 in donor cells leads to a favorable phenotype following

513 allotransplant, primarily through altered gene expression that leads to altered T cell function.

514

515

516

517 


\section{Discussion}

521

In this manuscript, as well as in our companion manuscript, we identified T Cell Factor-1 (TCF-

522

1) as a critical regulatory transcription factor for alloactivated mature T cells. TCF-1 is known to

523

be important for T cell development, as well as activation in some contexts (46) (47). However,

524 it was previously unknown whether TCF-1 played a role in regulating mature and alloactivated T

525 cells. Here, we show that loss of TCF-1 separates GVHD from GVL driven by CD8 T cells in a

526 clinically optimal fashion. While TCF-1 in donor T cells is dispensable for GVL effects, it is

527 essential for host tissue damage during GVHD. Importantly, we show here and in our companion

528 manuscript that the role of TCF-1 in regulating these T cells in unique in CD8 versus CD4 T

529 cells.

530 Using our unique mouse model with T cell-specific deletion of TCF-1 rather than global

531 deficiency, we were able to study mature CD8 T cells in an allo-HSCT model. This murine

532 model allows us to study T cell function, clinical outcomes, and gene expression all in one

533 model. Here, we showed that TCF-1-deficient donor CD8 T cells produce less severe and

534 persistent GVHD than WT CD8 T cells. Additionally, tissue damage in liver, skin, and small

535 intestine (all target organs) was reduced by loss of TCF-1 in donor CD8 T cells. This shows that

$536 \mathrm{TCF}-1$ in donor $\mathrm{T}$ cells is required for GVHD damage and persistence over time. Importantly,

537 GVL effects were not disrupted by loss of TCF-1, suggesting that TCF-1 is dispensable for GVL

538 effects. This was mostly likely due to an increase in perforin and granzyme B pre-transplant, as

539 well as increased granzyme B post-transplant for TCF-1-deficient CD8 T cells. Increased

540 expression of Eomes and T-bet may also have contributed to GVL effects $(10,16)(48)$. This

541 suggests that despite other functional changes which disrupt GVHD damage, the increase in

542 cytotoxic mediators helps maintain GVL effects driven by TCF cKO CD8 T cells. 
544 cytokine production - to better understand how TCF-1 regulates mature T cells to provide an

545 optimal disease phenotype. We showed that loss of TCF-1 in donor CD8 T cells leads to reduced

546 production of TNF- $\alpha$ (yet increased IFN- $\gamma$ production), increased predisposition to apoptosis,

547 increased exhaustion, and possibly reduced proliferation of alloactivated donor T cells. The

548 reduced survival of and production of TNF- $\alpha$ by donor CD8 T cells leads to the reduction in

549 GVHD severity. However, expression of perforin and Granyzme B were increased, allowing

550 these cells to maintain GVL effects despite their functional handicaps. In addition, although no

551 major migration defect was detected, loss of TCF-1 altered expression of chemokines and

552 chemokine receptors on donor CD8 T cells, both pre- and post-transplant. This phenotype caused

553 by loss of TCF-1 is clinically optimal, because it allows for clearance of residual malignant cells

554 while limiting the risk of life-threatening GVHD damage.

555 When we attempted to FACS-sort donor T cells from recipients at day 7, we found that

556 donor cell numbers for the TCF cKO-transplanted mice were significantly reduced in spleen and

557 trending towards reduced in liver. T cell survival must occur for persistence of GVHD

558 responses(36). These data support the idea that TCF-1-deficient donor cells are either dying or

559 not proliferating following transplant, which correlates with the observed increase in apoptotic

560 cells, as well as the reduction in disease severity beyond day 7 post-transplant. This observation,

561 coupled with the increase in exhaustion of TCF cKO donor CD8 T cells, suggests that donor

562 cells lacking TCF-1 are highly activated and cytotoxic to malignant cells early on following

563 transplant, but quickly die off or become exhausted, limiting GVHD progression. This also

564 makes sense given the observation that proliferation is higher in TCF cKO cells at day 7, because

565 we expect that these cells function normally or even better than WT cells, but then quickly lose 
566 functionality and die off. Again, this is critical as loss of TCF-1 allows clearance of tumor cells

567 through GVL effects, but after this point the donor cells no longer cause GVHD damage,

568 resolving disease quickly and with less tissue damage.

569 When we phenotyped TCF cKO and WT CD8 T cells prior to transplant, we found an 570 expansion of activated cells (CD122+, CD44+), as well as an increase in Eomes and T-bet 571 expression. Eomes and T-bet are both linked to expression of cytotoxic mediators, such as 572 perforin (Eomes) and IFN- $\gamma$ (T-bet) $(49,50)$. In addition, we found that CD8 central memory T

573 cells were expanded in TCF cKO mice. While effector and naive cells are known to cause severe 574 GVHD, memory cells are often associated with less severe disease $(10,32)$, suggesting that this 575 phenotypic change in TCF cKO cells may be beneficial for reducing disease severity. These 576 phenotypic effects were primarily cell-intrinsic, and driven by loss of TCF-1. We also found that 577 the suppressive profile of CD8 T cells is altered by loss of TCF-1. PD-1 expression was 578 decreased and PD-L1 expression was increased on TCF cKO CD8 donor T cells late after 579 transplant (day 14 and 21). This suggests that the donor cells may be less susceptible to 580 suppression by PD-L1+ cells (such as tumor cells), and may be more capable of suppressing 581 other nearby cells $(26,51)$. This could allow donor T cells lacking TCF-1 to attack tumor cells 582 but also suppress nearby alloactivated donor cells to limit disease.

583 Recent work has identified a CXCR5+ subset of CD8 T cells that arises following 584 chronic antigen stimulation. These cells are stem-like, and are thought to maintain the pool of 585 responding CD8 T cells during chronic immune responses (22, 23). Given that alloantigen is 586 constantly presented during GVHD, we sought to determine whether this cell subset also exists 587 in GVHD, and whether it may contribute to disease severity. Indeed, we found that CXCR5+ 588 CD8+ donor-derived T cells are found in mice allotransplanted with WT donor T cells. These 
589 mice develop severe GVHD and have poor survival. Interestingly, these cells appear to peak 590 around day 7 , which is typically the peak of disease severity by clinical scoring. We also found 591 that the frequency of these CXCR5+ CD8+ donor T cells is reduced in mice allotransplanted 592 with TCF cKO donor cells. TCF-1 is thought to be a critical factor for production of these cells 593 (52) yet modulation of TCF-1 in CD8 T cells only reduces the frequency of these cells.

594 Importantly, this reduction in CXCR5+ CD8+ T cells correlated with the pattern of reduced 595 disease severity and persistence in TCF cKO-transplanted mice. RNAseq results from D7 post596 transplant cells in spleen and liver show the same trend, with expression level of CXCR5 597 trending towards decreased in TCF cKO donor CD8 T cells. This suggests that CXCR5+ CD8+ 598 T cells may be responsible for driving chronic responses to alloantigen during GVHD, and that 599 depletion of this subset may prevent or reduce GVHD severity and persistence. Critically, GVL 600 effects were maintained despite reduction in the frequency of these cells from TCF cKO donors, 601 suggesting that CXCR5+ CD8+ T cells may be important for GVHD, but not GVL effects.

602 Further modulation or depletion of this cell subset could therefore potentially separate GVHD 603 from GVL effects.

604 Finally, to identify the changes to the genetic program of donor T cells that occurred in 605 the absence of TCF-1, we performed RNA sequencing on pre- and post-transplant donor T cells. 606 We found that CD8 donor T cells clustered together both by strain and by time (pre- and post607 transplant), and that many genes were altered by TCF-1 deficiency. When comparing pre608 transplant donor cells, no genes came up as significantly different when using FDR $\leq 0.05$, most 609 likely meaning that donor cells from WT and TCF cKO mice are similar pre-transplant.

610 Therefore, $\mathrm{p}$-value $\leq 0.05$ was used to define significant genes for pathway analysis (for pre611 transplant only). This also suggests that the changes to Eomes and T-bet expression seen in 
612 phenotyping were due to post-transcriptional changes. In pre-transplant cells, TCF-1 deficiency

613 led to changes in cell adhesion, TCR and other signaling pathways, and T cell differentiation,

614 among others. Of note, post-transplant donor cells from both spleen and liver showed changes to

615 necroptosis, cell cycle, autophagy, and cell senescence pathways. This strongly supports our ex

616 vivo evidence that TCF cKO CD8 donor T cells are more prone to apoptosis, and therefore most

617 likely die off following allotransplant, allowing disease to resolve. In addition, multiple immune

618 signaling pathways such as NOD, NFKB, and TNF signaling were affected by loss of TCF-1.

619 Therefore, TCF-1 controls these immune-related genetic programs in mature and allaoctivated

620 CD8 T cells.

621 In our companion manuscript, we also address the role of TCF-1 in regulating

622 alloactivated CD4 T cells. From this work, we found that regulation of CD4 versus CD8 T cells

623 by TCF-1 is different, most likely due to the ability of TCF-1 to maintain lineage identity and

624 alter gene expression in these subsets. Overall, our data show that TCF-1 plays a direct role in

625 regulating mature CD4 and CD8 T cells during alloactivation. Loss of TCF-1 in CD8 T cells (as

626 well as CD4 T cells) leads to reduced severity and persistence of GVHD, but maintains CD8 T

627 cell-driven GVL effects. This may be due at least in part to reduction of CXCR5+ CD8+ donor-

628 derived T cells, which correlate temporally with peak disease severity. Therefore, modulation of

629 TCF-1, and possibly of CXCR5+ CD8 T cells, may separate GVHD from GVL to provide

630 clinical benefit for allo-HSCT patients, or in treatment of other T cell-mediated diseases.

631

632

633

634

635

636

637

638 
650

651

652 653 654 655 656 657 658 659 660 661 662

\section{Materials and Methods}

Mouse Models. For transplant, the following female donor mice were used: B6-Ly5.1 (CD45.1+, “WT” or B6.SJL-Ptprca Pepcb/BoyCrl, 494 from Charles River), C57B1/6J (CD45.2+, "WT”, 000664 from Jackson Laboratories), Tcf7 flox x CD4cre (referred to here as “TCF cKO” (17), obtained from Dr. Jyoti Misra Sen at NIH by permission of Dr. Howard Xue, and bred in-house), or CD4cre (022071 from Jackson Laboratories, bred in-house). These donor mice were agematched to each other and to recipients as closely as possible. BALB/c female mice (CR:028 from Charles River, age 8 weeks or older) were used as recipient mice for transplant experiments, and Thy1.1 mice (B6.PL-Thy1a/CyJ, 000406 from Jackson Labs) were used as recipient mice for chimera experiments.

Allotransplant and Tumor Models. BALB/c recipient mice were irradiated with two doses of 400 cGy of x-rays (total dose 800 cGy), and rested for at least 12 hours between doses. Mice were also rested for 4 hours prior to transplantation. T cells (total CD3+ or CD8+) were separated from WT, CD4cre, or TCF cKO spleens using CD90.2 or CD8 microbeads and LS columns (Miltenyi, CD8: 130-117-044, CD90.2: 130-121-278, LS: 130-042-401). 1X106 donor cells were injected IV into the tail vein in PBS, along with $10 \times 10^{6} \mathrm{BALB} / \mathrm{c}$ bone marrow cells. Bone marrow was T-cell depleted with CD90.2 MACS beads (130-121-278 from Miltenyi) and LD columns (130-042-901 from Miltenyi). For short-term experiments, at day 7 post-transplant, recipient mice were euthanized and serum, spleen, lymph nodes, small intestine, or liver were collected, depending on the experiment. For migration assay, a 1:1 ratio of WT (CD45.1):WT(CD45.2) or WT (CD45.1):TCF cKO (CD45.2) donor cells was used to make up the $1 \mathrm{X} 10^{6} \mathrm{~T}$ cells. For GVHD and GVL experiments, recipient mice were also given $2 \mathrm{X} 10^{6} \mathrm{~B}-$ 
663 cell acute lymphoblastic leukemia (B-ALL) tumor cells expressing luciferase (10, 27). Recipient 664 mice were weighed, given a clinical score, and imaged using the IVIS 200 imaging system three

665 times per week until day 60 or longer. Clinical scores were composed of scores for skin integrity, 666 fur texture, posture, activity, diarrhea, and weight loss. Imaging was done by injecting recipients

667 i.p. with D-luciferin to detect tumor cell bioluminescence. To produce mixed bone marrow 668 chimeras, Thy1.1 mice were lethally irradiated and reconstituted with a 1:4 (WT:TCF cKO) 669 mixture of bone marrow cells (total $50 \times 10^{6}$ cells), then rested for 9 weeks. At 9 weeks, tail vein 670 blood was collected and checked by flow cytometry for CD45.1 and CD45.2 to ensure 671 reconstitution with both donor cell types. At 10 weeks, mice were used for phenotyping 672 experiments.

673

674 Flow Cytometry, Sorting, and Phenotyping. Splenocytes (or cells from other organs) were 675 obtained from WT or TCF cKO mice or recipient allotransplanted mice. Lymphocytes were 676 obtained and lysed with RBC Lysis Buffer (00-4333-57 from eBioscience) to remove red blood 677 cells if needed. Cells were then stained with extracellular markers for $30 \mathrm{~min}$ on ice in MACS 678 buffer (1X PBS with EDTA and 4g/L BSA). If intracellular markers were used, the cells were 679 then fixed and permeabilized using the Fix/Perm Concentrate and Fixation Diluent from FOXP3 680 Transcription Factor Staining Buffer Set (eBioscience cat. No. 00-5523-00). The cells were then 681 run on a BD LSR Fortessa cytometer and data was analyzed using FlowJo software v9 682 (Treestar). All antibodies were used at a 1:100 dilution. For FACS sorting, the same methods 683 was applied, and cells were run on a BD FACS Aria IIIu with cold-sorting blocks. Cells were 684 sorted into sorting media (50\% FBS in RPMI) or Trizol, depending on the experiment.

685 Depending on the experiment, antibodies used were: anti-CD4 (FITC, PE, BV785, BV21), anti- 
686

687

688

689

690

691

692

693

694

695

696

697

698

699

700

701

702

703

704

705

706

707

708

CD8 (FITC, PE, APC, PerCP, Pacific Blue, PE/Cy7), anti-CD3 (BV605 or APC/Cy7), antiH2Kb-Pacific Blue, anti-H2Kd-PE/Cy7, anti-CD122 (FITC or APC), anti-CD44 (APC or Pacific Blue), anti-CD62L (APC/Cy7), anti-TNF- $\alpha$-FITC, anti-IFN- $\gamma$-APC, anti-Eomes (AF488 or PE/Cy7), anti-T-bet-BV421, anti-CD45.2-PE/Dazzle594, anti-CD45.1-APC, anti-Ki67 (PE or BV421), anti-PD-1-BV785, anti-CTLA-4-PE, and anti-PD-L1 (PE or PE/Dazzle594), antiAnnexin V-FITC, LIVE/DEAD Near IR, anti-EdU-AF647 (click chemistry kit), anti-TOX-APC, and anti-CXCR5-APC.

Histology. Recipient mice were allotransplanted as described, and organs were removed for histology at day 7, day 14, and day 21 post-transplant. Spleen, liver, small intestine, and skin (from ear and back) were fixed, sectioned, and stained with H\&E at Cornell University ( https://www.vet.cornell.edu/animal-health-diagnostic-center/laboratories/anatomic-

pathology/services). A pathologist (A.M.) who was blinded to slide identity and study conditions then graded the sections for GVHD damage on a scale of 0-4. Criteria were: for skin - basal cell vacuolation up to frank epidermal denudation, for small intestine - single cell necrosis up to diffuse mucosal denudation, and for liver - percent of bile duct showing epithelial damage (I $\leq 25 \%$, II $25-49 \%$, III 50-74\%, IV 75-100\%).(53)(54).

Isolation of lymphocytes from liver, small intestine, or lymph nodes. Livers were perfused with 5-10mL of ice cold PBS to remove RBCs, then smashed through a 70uM filter with PBS. Cells were centrifuged to remove debris, and then spun for 22 minute spin in $40 \%$ Percoll in RPMI/PBS (22 degrees C, 2200rpm, no brake, no acceleration) to isolate lymphocytes. RBC Lysis Buffer was then used to remove any remaining RBCs, and cells were put into MACS 
709 buffer (BSA in PBS). To obtain lymphocytes from gut, small intestine was removed to ice cold

710 MACS buffer, cut open, and washed with MACS. Strip buffer (1XPBS, FBS, EDTA 0.5M, and

711 DTT 1M) was used to remove epithelium (shaking for $30 \mathrm{~min}$ at $37 \mathrm{C}$ ), followed by mincing and

712 incubation with digestion buffer (collagenase, DNAse, and RPMI) for $30 \mathrm{~min}$ at $37 \mathrm{C}$ (while

713 shaking). The gut pieces were vortexed and mashed through a 70uM filter with the liquid to

714 obtain a cell suspension. Percoll was used as for liver to isolate lymphocytes from this

715 suspension, but RBC lysis was not done. For lymph nodes, the inguinal, brachial, and axillary

716 lymph nodes were taken from recipients and ground between glass slides. Cells were washed

717 into a tube with MACS and spun to remove debris.

718

719 Cytokine Restimulation. Recipient BALB/c mice were allotransplanted with $1.5 \mathrm{X} 10^{6}$ CD3 donor

720 T cells and $10 \times 10^{6} \mathrm{BALB} / \mathrm{c} \mathrm{BM}$, and euthanized at day 7. Splenocytes were taken and cultured

721 for 6 hours with GolgiPlug (1:1000) and PBS (control) or anti-CD3 (1ug/mL)/anti-CD28

$722(2 \mathrm{ug} / \mathrm{mL})(\mathrm{TCR}$ stimulation) at $37 \mathrm{C}$ and $7 \% \mathrm{CO} 2$. After 6 hours of culture, the cells were

723 stained for CD3, CD4, CD8, H2Kb, TNF- $\alpha$, and IFN- $\gamma$ using the BD Cytokine Staining kit (BD

724 Biosciences, 555028), and run on a flow cytometer.

725

726 LegendPLEX Serum ELISA Assay. Serum from cardiac blood was collected from recipient

727 mice in the cytokine restimulation experiment. Serum was analyzed using the Biolegend

728 LEGENDPlex Assay Mouse Th Cytokine Panel kit (741043). This kit quantifies serum

729 concentrations of: IL-2 (T cell proliferation), IFN-y and TNF-a (Th1 cells, inflammatory), IL-4,

730 IL-5, and IL-13 (Th2 cells), IL-10 (Treg cells, suppressive), IL-17A/F (Th17 cells), IL-21 (Tfh 
731 cells), IL-22 (Th22 cells), IL-6 (acute/chronic inflammation/T cell survival factor), and IL-9

732 (Th2, Th17, iTreg, Th9 - skin/allergic/intestinal inflammation).

733

734 Western blot. Splenocytes from WT or TCF cKO donor mice, or from WT- or TCF cKO-

735 transplanted recipient mice as described $\left(1 \mathrm{X} 10^{6} \mathrm{CD} 8\right.$ donor T cells and $\left.10 \mathrm{X} 10^{6} \mathrm{BALB} / \mathrm{c} \mathrm{BM}\right)$,

736 were collected. CD8+ T cells were separated using CD8 MACS beads. These cells were counted

737 and lysed with RIPA Buffer (89900 from Thermo Fisher) plus protease inhibitors (11697498001

738 from Millipore Sigma) to obtain lysates. The lysates were run on a Western blot and probed for

739 perforin, Granzyme B, and actin.

740

741 Migration Assay. Recipient BALB/c mice were allotransplanted as before with $10 \mathrm{X} 10^{6} \mathrm{BALB} / \mathrm{c}$

$742 \mathrm{BM}$ and $1 \mathrm{X} 10^{6}$ donor CD3 T cells. However, the $1 \times 10^{6}$ donor T cells were a 1:1 mixture of WT

743 CD45.1+ CD3 T cells with either WT CD45.2+ cells or TCF cKO CD45.2+ cells. Prior to

744 transplant, the donor cells were checked to ensure a 1:1 of CD4/CD8 T cells and of donor strain

745 (WT:WT or WT:TCF cKO). 7 days post-transplant, recipients were euthanized and the spleen,

746 lymph nodes, liver, and small intestine were removed. Lymphocytes were obtained from the

747 organs as described, and stained for CD3, CD4, CD8, H2Kb, H2Kd, CD45.1, and CD45.2. The

748 ratio of CD45.1 to CD45.2 cells was determined from flow cytometry data (i.e. WT to WT or

749 WT to TCF cKO).

750

751 qPCR Analysis. To perform qPCR, BALB/c mice were allotransplanted as described (1X10

$752 \mathrm{CD} 3$ donor T cells and $10 \times 10^{6} \mathrm{BALB} / \mathrm{c} \mathrm{BM}$ ). Pre-transplant donor cells and post-transplant (day

753 7) spleen and liver cells from recipients were FACS-sorted to obtain CD8+ donor cells. The cells 
754 were sorted into Trizol, RNA was extracted using chloroform

755 (https://www.nationwidechildrens.org/ Document/Get/93327), and eluted using the Qiagen

756 RNEasy Minikit (74104 from Qiagen). Concentration was checked with a spectrophotometer,

757 then RNA was converted to cDNA with an Invitrogen Super Script IV First Strand Synthesis

758 System kit (18091050 from Invitrogen). Final cDNA concentration was checked with a

759 spectrophotometer, then cDNA was mixed with Taqman Fast Advanced Master Mix (4444557

760 from Invitrogen) at a 10ng/uL cDNA concentration. This master mix was added to premade 96

761 well TaqMan Array plates with chemokine/chemokine receptor primers (Thermo Fisher, Mouse

762 Chemokines \& Receptors Array plate, 4391524). qPCR was performed in a Quant Studio 3

763 thermocycler, and data were analyzed using the Design and Analysis software v2.4 (provided by

764 Thermo Fisher). Five separate recipient mice were sorted and cells were combined to make one

765 sample for qPCR testing per condition/organ.

766

767 Proliferation Assay. Recipient BALB/c mice were allotransplanted as before (1X10 ${ }^{6}$ CD3 donor

$768 \mathrm{~T}$ cells and $\left.10 \mathrm{X} 10^{6} \mathrm{BALB} / \mathrm{c} \mathrm{BM}\right)$. On day 5 and 6 post-transplant, recipient mice were injected

769 i.p. with $25 \mathrm{mg} / \mathrm{kg}$ of EdU in PBS. At day 7, recipient mice were euthanized and spleen and liver

770 were processed to obtain lymphocytes. An EdU click chemistry kit (C10424 from Invitrogen)

771 was used along with antibodies for $\mathrm{CD} 3, \mathrm{CD} 4, \mathrm{CD} 8$, and $\mathrm{H} 2 \mathrm{~Kb}$ to detect proliferating donor

772 cells. In vitro culture attempts mentioned as empirical observations were done at $37 \mathrm{C}, 7 \% \mathrm{CO} 2$,

773 with anti-CD3 (1ug/mL), anti-CD28 (2ug/mL), and in some cases, IL-2 (1000IU/mL).

774

775 Death Assay. To assess apoptosis and death in donor cells, recipient mice were allotransplanted

776 as above $\left(1 \times 10^{6} \mathrm{CD} 3\right.$ donor T cells and $\left.10 \times 10^{6} \mathrm{BALB} / \mathrm{c} \mathrm{BM}\right)$. At 7 days post-transplant, 
777 recipient mice were euthanized and spleen and liver were taken. Lymphocytes were obtained as

778 described and stained with antibodies for $\mathrm{CD} 3, \mathrm{CD} 4, \mathrm{CD} 8$, and $\mathrm{H} 2 \mathrm{~Kb}$ to identify donor cells.

779 The cells were also stained with Annexin V-FITC (V13242 from Invitrogen) and LIVE/DEAD

780 Near IR (L34976 from Invitrogen). Annexin V and NIR were used to identify dead

781 (Ann.V+NIR+), live (Ann.V-NIR-), and apoptotic (Ann.V+NIR-) cells.

782

783 Exhaustion/Activation Assay. To assess exhaustion and activation in donor cells, recipient mice

784 were allotransplanted as before $\left(1 \times 10^{6} \mathrm{CD} 3\right.$ donor T cells and $\left.10 \times 10^{6} \mathrm{BALB} / \mathrm{c} \mathrm{BM}\right)$ and

785 euthanized at day 7. Lymphocytes were obtained from spleen and liver, and stained for CD3,

$786 \mathrm{CD} 4, \mathrm{CD} 8, \mathrm{H} 2 \mathrm{~Kb}$, TOX, and Ki67.

787

788 Time course Assay. Recipient mice were allotransplanted as before (1X10 CD8 donor T cells

789 and $\left.10 \times 10^{6} \mathrm{BALB} / \mathrm{c} \mathrm{BM}\right)$, and euthanized at day 7 , day 14 , or day 21 in groups. Spleen and liver

790 were taken from each mouse, and lymphocytes were isolated. The cells were stained for CXCR5,

791 PD-1, PD-L1, CTLA-4, CD3, CD4, CD8, and H2Kb.

792

793 DNA Extraction and PCR. Donor mice were genotyped using PCR on DNA extracted from ear

794 punches. At 4 weeks of age mice were ear punched, and DNA was extracted using the Accustart

795 II Mouse Genotyping kit (95135-500 from Quanta Biosciences). Standard PCR reaction

796 conditions and primer sequences from Jackson Laboratories were used for Eomes, T-bet, and

797 CD4cre. For Tcf7, primer sequences and reaction conditions were obtained from Dr. Jyoti Misra

798 Sen of NIH.

799 
800 RNA Sequencing. Recipient mice were allotransplanted as before (1X10 ${ }^{6} \mathrm{CD} 3$ donor T cells and $80110 \times 10^{6} \mathrm{BALB} / \mathrm{c} \mathrm{BM}$ ), except that donor CD8 T cells were also FACS-sorted prior to transplant.

802 A sample of sorted donor cells was saved for RNA sequencing. At day 7 post-transplant, donor

803 CD8 T cells were FACS-sorted back from recipient spleen and liver, and for TCF cKO-

804 transplanted mice, cells were also collected at day 14. The cells were all sorted into Trizol, then

805 RNA was extracted and prepped by the Molecular Analysis Core (SUNY Upstate,

806 https://www.upstate.edu/research/facilities/molecular-analysis.php)._Paired end sequencing was

807 done with an Illumina NovaSeq 6000 system at the University at Buffalo Genomics Core

808 (http://ubnextgencore.buffalo.edu). Data were provided and analyzed in Partek Flow. Data will

809 be deposited (https://www.ncbi.nlm.nih.gov/geo/).

810

811 Statistical Analysis. Unless otherwise noted in the figure legends, all numerical data are

812 presented as means and standard deviations with or without individuals points. Analysis was

813 done in GraphPad Prism v7 or v9. Most data were analyzed with Student's t-test, one-way

814 ANOVA, or two-way ANOVA, with Tukey's multiple comparisons test for ANOVA methods,

815 depending on the number of groups. Chi-square analyses were done for GVHD histology grades.

816 Kaplan-Meier survival analyses were done for survival experiments. All tests were two-sided,

817 and p-values less than or equal to $(\leq) 0.05$ were considered significant. Transplant experiments

818 used 3-5 mice per group, with at least two repeats. Ex vivo experiments were done two to three

819 times unless otherwise noted with at least three replicates per condition each time. RNAseq was

820 done once with three replicates per group and condition. qPCR was done once with one sample

821 per condition, and 5 mice combined to make the one sample. Sorting was done once for each of

822 these two experiments, and data were recorded from one experiment for Supp. Fig. 3. The time 
823 course assay was done once with 4-6 samples per group. Western blots were done twice for pre-

824 transplant and once for post-transplant, one experiment each is shown.

825

826 Study Approval. All animal studies were reviewed and approved by the IACUC at SUNY

827 Upstate Medical University. All procedures and experiments were performed according to these

828 approved protocols.

829

830

831

832

833

834

835

836

837

838

839

840

841

842

843

844

845 


\section{Author Contributions}

847 RH designed and conducted experiments, analyzed data, and wrote the manuscript. MK assisted

848 with scientific/technical research design. MK and JMS edited the manuscript. MK and MM

849 assisted with data collection. AM performed histology analyses. QY provided technical and 850 scientific advice and assisted with data analysis. IF produced gene expression heatmaps for

851 RNAseq data.

852

853 Acknowledgments

854 We thank all members of the Karimi lab for helpful discussions. This research was funded in part 855 by a grant from the National Blood Foundation Scholar Award to MK, the National Institutes of 856 Health (NIH LRP \#L6 MD0010106 and K22 (AI130182) to MK), and an Upstate Medical

857 University Cancer Center grant (1146249-1-75632) to MK. JMS was supported by the

858 Intramural Research Program of the National Institute of Aging. We thank Dr. Howard Xue for

859 permission to use TCF cKO mice. TCF cKO mice were provided by Dr. Jyoti Misra Sen from

860 NIH. Flow cytometers and sorters were provided and maintained by Lisa Phelps of the SUNY

861 Upstate Flow Core. RNA extraction and prep was done by Karen Gentile of the SUNY Upstate

862 Molecular Analysis core, and RNA sequencing was done by the University at Buffalo Genomics

863 Core.

864

865

866

867

868

869

870

871

872

873 
874

875

876

877

878

879

880

881

882

883

884

885

886

887

888

889

890

891

892

893

894

895

896

897

898

899

900

901

902

903

904

905

906

907

908

909

910

911

912

913

914

915

916

917

\section{References}

1. Breems DA, and Lowenberg B. Autologous stem cell transplantation in the treatment of adults with acute myeloid leukaemia. Br J Haematol. 2005;130(6):825-33.

2. Blazar BR, Murphy WJ, and Abedi M. Advances in graft-versus-host disease biology and therapy. Nat Rev Immunol. 2012;12(6):443-58.

3. Ferrara JL, Levine JE, Reddy $P$, and Holler E. Graft-versus-host disease. Lancet. 2009;373(9674):1550-61.

4. Ferrara JL. Blood and Marrow Transplant Clinical Trials Network: Progress since the State of the Science Symposium 2007. Biology of blood and marrow transplantation : journal of the American Society for Blood and Marrow Transplantation. 2014;20(2):14953.

5. Dickinson AM, Norden J, Li S, Hromadnikova I, Schmid C, Schmetzer H, et al. Graftversus-Leukemia Effect Following Hematopoietic Stem Cell Transplantation for Leukemia. Front Immunol. 2017;8:496.

6. Murray J, Stringer J, and Hutt D. In: Kenyon M, and Babic A eds. The European Blood and Marrow Transplantation Textbook for Nurses: Under the Auspices of EBMT. Cham (CH); 2018:221-51.

7. Bell GM, Anderson AE, Diboll J, Reece R, Eltherington O, Harry RA, et al. Autologous tolerogenic dendritic cells for rheumatoid and inflammatory arthritis. Ann Rheum Dis. 2017;76(1):227-34.

8. Gam R, Shah P, Crossland RE, Norden J, Dickinson AM, and Dressel R. Genetic Association of Hematopoietic Stem Cell Transplantation Outcome beyond Histocompatibility Genes. Front Immunol. 2017;8:380.

9. Ghimire S, Weber D, Mavin E, Wang XN, Dickinson AM, and Holler E. Pathophysiology of GvHD and Other HSCT-Related Major Complications. Front Immunol. 2017;8:79.

10. Mammadli $M$, Huang W, Harris R, Sultana A, Cheng $Y$, Tong W, et al. Targeting Interleukin-2-Inducible T-Cell Kinase (ITK) Differentiates GVL and GVHD in Allo-HSCT. Front Immunol. 2020;11:593863.

11. Bleakley $M$, Heimfeld S, Jones LA, Turtle C, Krause D, Riddell SR, et al. Engineering human peripheral blood stem cell grafts that are depleted of naive $T$ cells and retain functional pathogen-specific memory T cells. Biology of blood and marrow transplantation : journal of the American Society for Blood and Marrow Transplantation. 2014;20(5):705-16.

12. Sharma A, Berga-Bolanos R, and Sen JM. T cell factor-1 controls the lifetime of CD4+ $\mathrm{CD} 8+$ thymocytes in vivo and distal $T$ cell receptor alpha-chain rearrangement required for NKT cell development. PLoS One. 2014;9(12):e115803.

13. Germar K, Dose M, Konstantinou T, Zhang J, Wang H, Lobry C, et al. T-cell factor 1 is a gatekeeper for T-cell specification in response to Notch signaling. Proc Natl Acad Sci U S A. 2011;108(50):20060-5.

14. Ma J, Wang R, Fang X, and Sun Z. beta-catenin/TCF-1 pathway in T cell development and 
918

919

920

921

922

923

924

925

926

927

928

929

930

931

932

933

934

935

936

937

938

939

940

941

942

943

944

945

946

947

948

949

950

951

952

953

954

955

956

957

958

959

960

961

differentiation. J Neuroimmune Pharmacol. 2012;7(4):750-62.

15. Paley MA, and Wherry EJ. TCF-1 flips the switch on Eomes. Immunity. 2010;33(2):145-7.

16. Fu J, Wang D, Yu Y, Heinrichs J, Wu Y, Schutt S, et al. T-bet is critical for the development of acute graft-versus-host disease through controlling $T$ cell differentiation and function. J Immunol. 2015;194(1):388-97.

17. Steinke FC, Yu S, Zhou X, He B, Yang W, Zhou B, et al. TCF-1 and LEF-1 act upstream of Th-POK to promote the CD4(+) T cell fate and interact with Runx3 to silence $\mathrm{Cd} 4$ in CD8(+) T cells. Nat Immunol. 2014;15(7):646-56.

18. Bouazzaoui A, Spacenko E, Mueller G, Miklos S, Huber E, Holler E, et al. Chemokine and chemokine receptor expression analysis in target organs of acute graft-versus-host disease. Genes Immun. 2009;10(8):687-701.

19. Henden AS, and Hill GR. Cytokines in Graft-versus-Host Disease. J Immunol. 2015;194(10):4604-12.

20. Hill GR, and Koyama M. Cytokines and costimulation in acute graft-versus-host disease. Blood. 2020;136(4):418-28.

21. Zhang J, Chen HM, Ma G, Zhou Z, Raulet D, Rivera AL, et al. The mechanistic study behind suppression of GVHD while retaining GVL activities by myeloid-derived suppressor cells. Leukemia. 2019;33(8):2078-89.

22. Kircher B, Schumacher P, and Nachbaur D. Granzymes A and B serum levels in allo-SCT. Bone Marrow Transplant. 2009;43(10):787-91.

23. Valentine KM, and Hoyer KK. CXCR5+ CD8 T Cells: Protective or Pathogenic? Front Immunol. 2019;10:1322.

24. Im SJ, Konieczny BT, Hudson WH, Masopust D, and Ahmed R. PD-1+ stemlike CD8 T cells are resident in lymphoid tissues during persistent LCMV infection. Proc Natl Acad Sci U S A. 2020;117(8):4292-9.

25. Shen $\mathrm{Y}, \mathrm{Qu} \mathrm{QX}$, Jin MN, and Chen C. Investigating the role of circulating CXCR5expressing CD8+ T-cells as a biomarker for bacterial infection in subjects with pneumonia. Respir Res. 2019;20(1):54.

26. Flowers ME, and Martin PJ. How we treat chronic graft-versus-host disease. Blood. 2015;125(4):606-15.

27. Cheng $\mathrm{Y}$, Chikwava $\mathrm{K}, \mathrm{Wu} \mathrm{C}$, Zhang H, Bhagat A, Pei D, et al. LNK/SH2B3 regulates IL-7 receptor signaling in normal and malignant B-progenitors. $J$ Clin Invest. 2016;126(4):1267-81.

28. Cooke KR, Kobzik L, Martin TR, Brewer J, Delmonte J, Jr., Crawford JM, et al. An experimental model of idiopathic pneumonia syndrome after bone marrow transplantation: I. The roles of minor H antigens and endotoxin. Blood. 1996;88(8):32309.

29. Pidala J, Kim J, Anasetti C, Nishihori T, Betts B, Field T, et al. The global severity of chronic graft-versus-host disease, determined by National Institutes of Health consensus criteria, is associated with overall survival and non-relapse mortality. Haematologica. 2011;96(11):1678-84.

30. https://www.uptodate.com/contents/clinical-manifestations-diagnosis-and-grading-ofacute-graft-versus-host-disease

31. Sun R, Wu Y, Zhou H, Wu Y, Yang Z, Gu Y, et al. Eomes Impedes Durable Response to 
962

963

964

965

966

967

968

969

970

971

972

973

974

975

976

977

978

979

980

981

982

983

984

985

986

987

988

989

990

991

992

993

994

995

996

997

998

999

1000

1001

1002

1003

1004

1005

Tumor Immunotherapy by Inhibiting Stemness, Tissue Residency, and Promoting the Dysfunctional State of Intratumoral CD8(+) T Cells. Front Cell Dev Biol. 2021;9:640224.

32. Dutt S, Baker J, Kohrt HE, Kambham N, Sanyal M, Negrin RS, et al. CD8+CD44(hi) but not CD4+CD44(hi) memory $T$ cells mediate potent graft antilymphoma activity without GVHD. Blood. 2011;117(11):3230-9.

33. Du W, and Cao X. Cytotoxic Pathways in Allogeneic Hematopoietic Cell Transplantation. Front Immunol. 2018;9:2979.

34. Beilhack A, Schulz S, Baker J, Beilhack GF, Wieland CB, Herman El, et al. In vivo analyses of early events in acute graft-versus-host disease reveal sequential infiltration of T-cell subsets. Blood. 2005;106(3):1113-22.

35. Paczesny S, Hanauer D, Sun Y, and Reddy P. New perspectives on the biology of acute GVHD. Bone Marrow Transplant. 2010;45(1):1-11.

36. Chakraverty $\mathrm{R}$, and Sykes $\mathrm{M}$. The role of antigen-presenting cells in triggering graftversus-host disease and graft-versus-leukemia. Blood. 2007;110(1):9-17.

37. Ioannidis $\mathrm{V}$, Beermann $\mathrm{F}$, Clevers $\mathrm{H}$, and Held $\mathrm{W}$. The beta-catenin--TCF-1 pathway ensures CD4(+)CD8(+) thymocyte survival. Nat Immunol. 2001;2(8):691-7.

38. Xu Z, Xing S, Shan Q, Gullicksrud JA, Bair TB, Du Y, et al. Cutting Edge: beta-CateninInteracting Tcf1 Isoforms Are Essential for Thymocyte Survival but Dispensable for Thymic Maturation Transitions. J Immunol. 2017;198(9):3404-9.

39. Riley JL. PD-1 signaling in primary T cells. Immunol Rev. 2009;229(1):114-25.

40. Fife BT, and Pauken KE. The role of the PD-1 pathway in autoimmunity and peripheral tolerance. Ann N Y Acad Sci. 2011;1217:45-59.

41. Francisco LM, Sage PT, and Sharpe AH. The PD-1 pathway in tolerance and autoimmunity. Immunol Rev. 2010;236:219-42.

42. Diskin B, Adam S, Cassini MF, Sanchez G, Liria M, Aykut B, et al. PD-L1 engagement on T cells promotes self-tolerance and suppression of neighboring macrophages and effector T cells in cancer. Nat Immunol. 2020;21(4):442-54.

43. Walker LS, and Sansom DM. Confusing signals: recent progress in CTLA-4 biology. Trends Immunol. 2015;36(2):63-70.

44. Im SJ, Hashimoto M, Gerner MY, Lee J, Kissick HT, Burger MC, et al. Defining CD8+ T cells that provide the proliferative burst after PD-1 therapy. Nature. 2016;537(7620):417-21.

45. https://link.springer.com/article/10.1007/s11515-017-1445-3

46. Yu S, Zhou X, Steinke FC, Liu C, Chen SC, Zagorodna O, et al. The TCF-1 and LEF-1 transcription factors have cooperative and opposing roles in T cell development and malignancy. Immunity. 2012;37(5):813-26.

47. https://link.springer.com/article/10.1007/s11515-017-1445-3

48. Fu J, Wu Y, Nguyen H, Heinrichs J, Schutt S, Liu Y, et al. T-bet Promotes Acute Graftversus-Host Disease by Regulating Recipient Hematopoietic Cells in Mice. J Immunol. 2016;196(7):3168-79.

49. Lord GM, Rao RM, Choe $H$, Sullivan BM, Lichtman AH, Luscinskas FW, et al. T-bet is required for optimal proinflammatory CD4+ T-cell trafficking. Blood. 2005;106(10):34329.

50. Pearce EL, Mullen AC, Martins GA, Krawczyk CM, Hutchins AS, Zediak VP, et al. Control of effector CD8+ T cell function by the transcription factor Eomesodermin. Science. 
2003;302(5647):1041-3.

1008

51. Chen Y, Yu M, Zheng Y, Fu G, Xin G, Zhu W, et al. CXCR5(+)PD-1(+) follicular helper CD8 T

1009 cells control B cell tolerance. Nat Commun. 2019;10(1):4415.

1010

52. https://link.springer.com/article/10.1007/s11515-017-1445-3

1011

53. http://surgpathcriteria.stanford.edu/transplant/skinacutegvhd/printable.html

54. http://surgpathcriteria.stanford.edu/transplant/giacutegvhd/printable.html

1012

1013

1014

1015

1016

1017

1018

1019

1020

1021

1022

1023

1024

1025 
CD8+ Donor T Cells

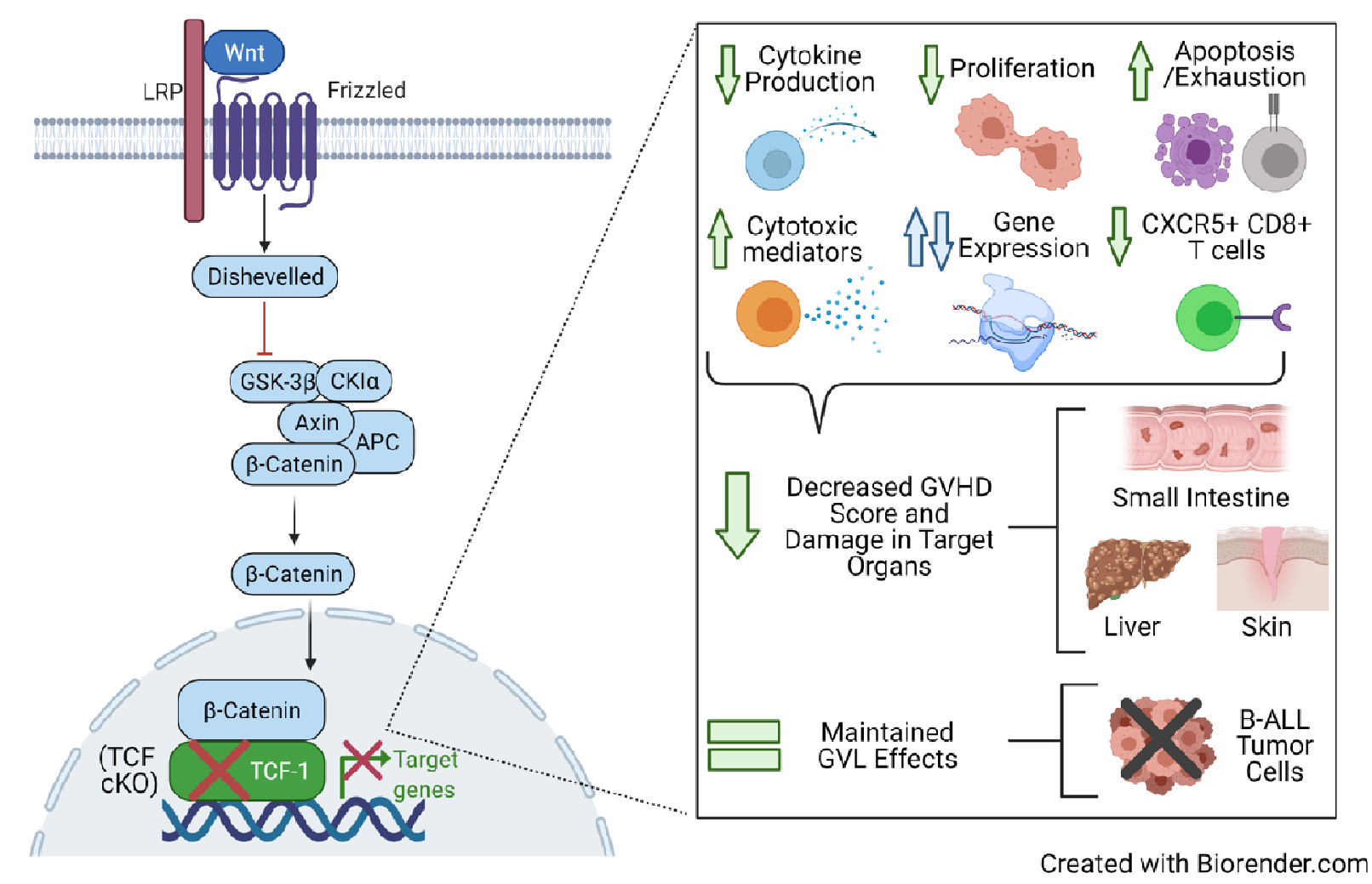

1028 Summary Figure: TCF-1 separates GVHD from GVL by regulating mature alloactivated

CD8 $\mathrm{T}$ cells. T Cell Factor-1 (TCF-1) is a T cell transcription factor involved in the Wnt pathway. Conditional deletion of TCF-1 in T cells led to reduced GVHD, yet maintained GVL effects again B-cell acute lymphoblastic leukemia (B-ALL) tumor cells. This occurred primarily due to: reduced cytokine production and proliferation; increased apoptosis, exhaustion, and production of cytotoxic mediators; and altered gene expression. The observed reduction in CXCR5+ CD8+ T cells may also have contributed to the reduced GVHD severity. These alterations led to reduced damage to target organs (liver, skin, and small intestine), yet the CD8 $\mathrm{T}$ cells were still able to clear tumor cells. 
1037

1038

1039

1040

1041

1042

1043

1044

1045

1046

1047

1048

1049

1050

1051

1052
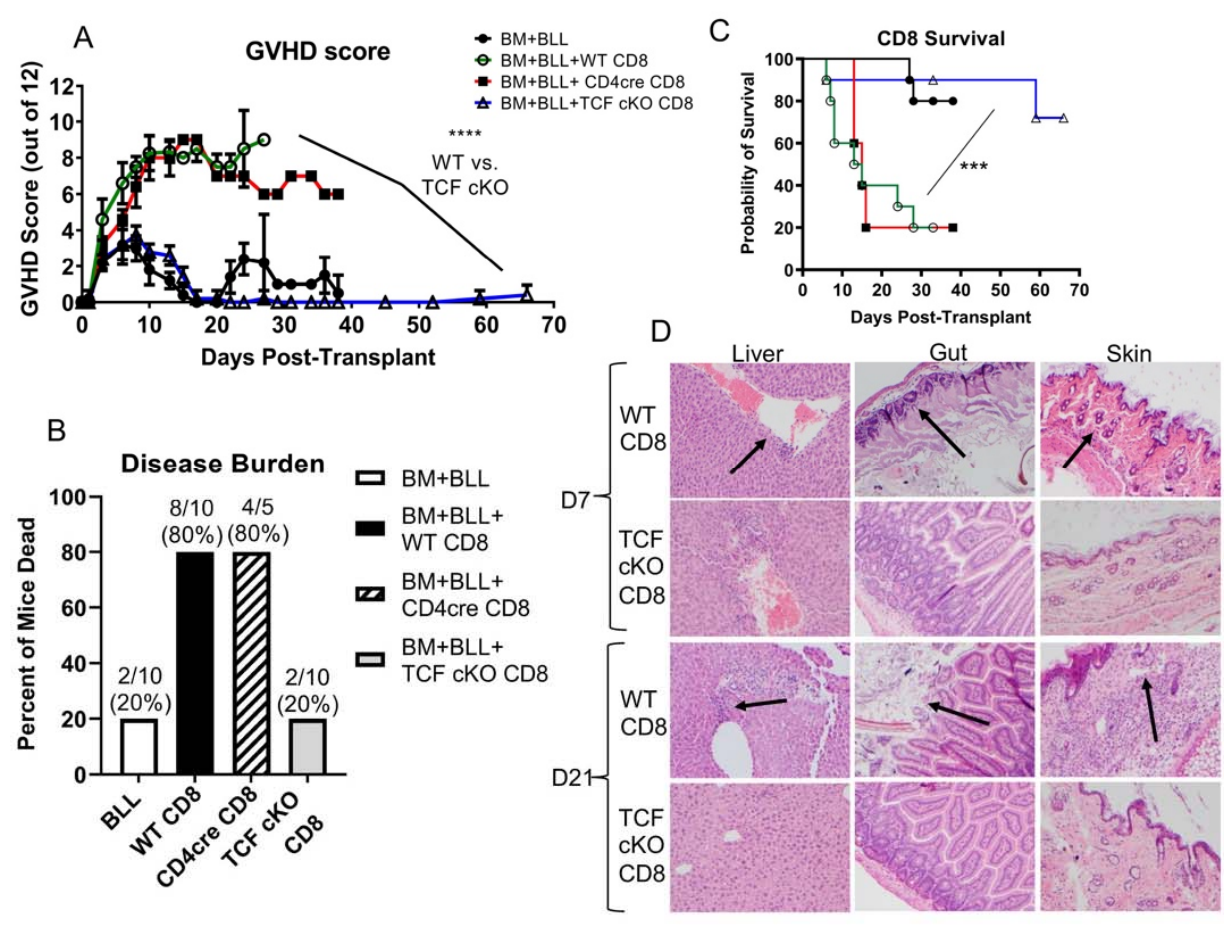
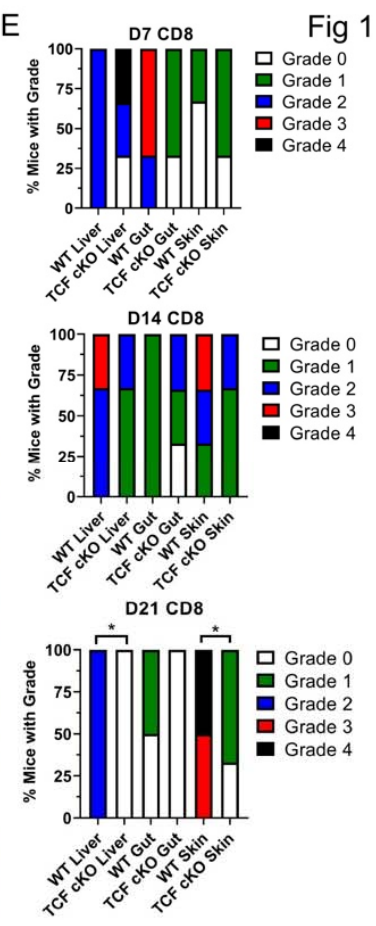

Figure 1: Loss of TCF-1 in donor CD8 T cells reduces severity and persistence of GVHD

symptoms. BALB/c recipient mice (MHC haplotype D) were lethally irradiated and allotransplanted with 1 X10 $0^{6} \mathrm{CD} 8 \mathrm{~T}$ cells from WT, CD4cre, or TCF cKO donor mice (MHC haplotype $\mathrm{b}$ ), as well as $10 \mathrm{X} 10^{6} \mathrm{~T}$ cell-depleted bone marrow cells (BM) from BALB/c mice (MHC haplotype d). Recipient mice were also given 2X10 $10^{5}$ luciferase-expressing B-ALL tumor cells $(10,40)$. (A) The recipient mice were given a GVHD clinical score three times per week until day 66, based on combined scores of fur texture, activity level, weight loss, posture, skin integrity, and diarrhea. Mean and SD are plotted, analyzed by two-way ANOVA. (B) Disease burden as determined by number of mice in each group that died by the end of the experiment.

Percentage and number of mice listed above the bar for each group. (C) Survival for each group of recipient mice over the 66 days post-transplant, analyzed by Kaplan-Meier survival analysis.

(D) At day 7, day 14, and day 21 post-transplant, organs were taken from recipient mice for histology analyses. Skin, liver, spleen, and small intestine were sectioned, stained with H\&E, and 
1053 graded by a pathologist (A.M.) blinded to study conditions and slide groups. Representative 1054 sections for each organ per group and timepoint are shown. Arrows indicate tissue damage or 1055 lymphocyte infiltration. (E) Histology grades for each group of mice, shown as percentage of 1056 mice per group experiencing that grade. Grades 3-4 are considered severe GVHD, while grades 1057 0-2 are considered mild GVHD. Data were analyzed by chi-square analysis. For all graphs, * 1058 means $\mathrm{p}$-value $\leq 0.05, * * *$ means $\mathrm{p}$-value $\leq 0.001$, and $* * * *$ means $\mathrm{p}$-value $\leq 0.0001 . \mathrm{N}=3-5$ per 1059 group for A-C with one representative experiment shown, N=3 per group for D-E, one 1060 representative photo shown and summary data in $\mathbf{E}$.
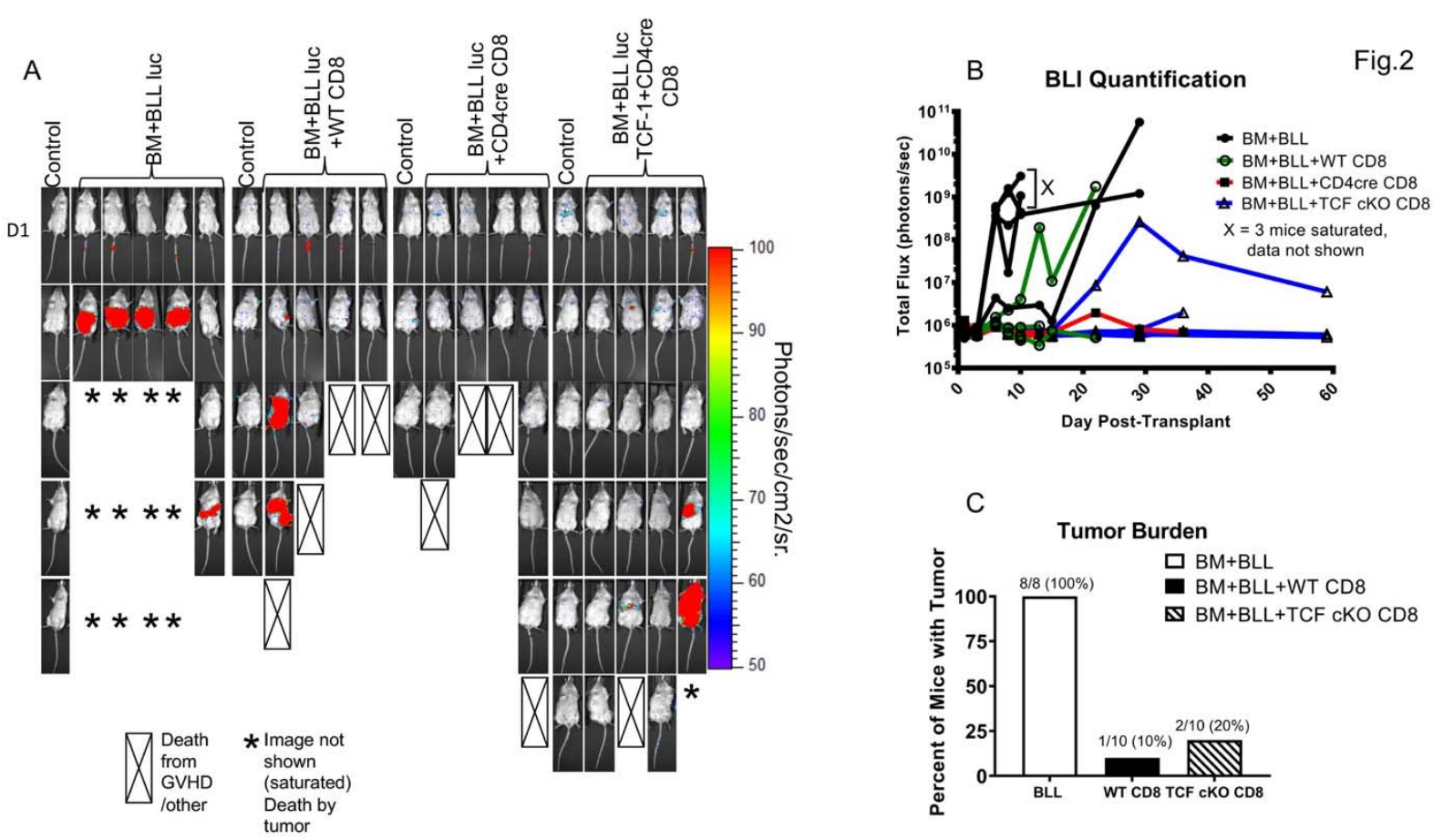

1062 Figure 2: TCF-1-deficient CD8 T cells retain anti-tumor capabilities for the graft-versus-

1063 leukemia (GVL) effect. BALB/c mice were allotransplanted as in Fig. 1 with T cell-depleted

$1064 \mathrm{BM}$ and WT, CD4cre, or TCF cKO CD8 donor T cells, along with $2 \mathrm{X} 10^{5}$ luciferase-expressing

1065 B-ALL tumor cells. Recipient mice were then injected with D-luciferin (i.p.) and imaged using

1066 the IVIS 200 imaging system. (A) Representative set of photos showing the tumor 
1067 bioluminescence and localization for transplanted groups. Control mice were untransplanted,

$1068 \mathrm{BM}+\mathrm{BLL}$ mice received only BM and tumor cells, while the other groups received BM, tumor

1069 cells, and either WT, CD4cre, or TCF cKO donor CD8 T cells. Boxes with an "X" indicate death

1070 from GVHD or tumor, * indicates that the image was saturated due to excessively high signal, so

1071 the image is not shown. (B) Quantification of bioluminescence signal for each mouse in the

1072 transplanted groups for one representative experiment, as determined by total flux (photons per

1073 second). Note: 3 mice from the BM+BLL luc group in this experiment had saturated signals, so

1074 the data was excluded. (C) Tumor burden for tumor only, tumor with WT CD8, and tumor with

1075 TCF cKO CD8 groups, as determined by number/percentage of mice showing tumor

1076 bioluminescence and tumor presence at the end of the experiment. $\mathrm{N}=3-5$ per group with one

1077 representative experiment shown.
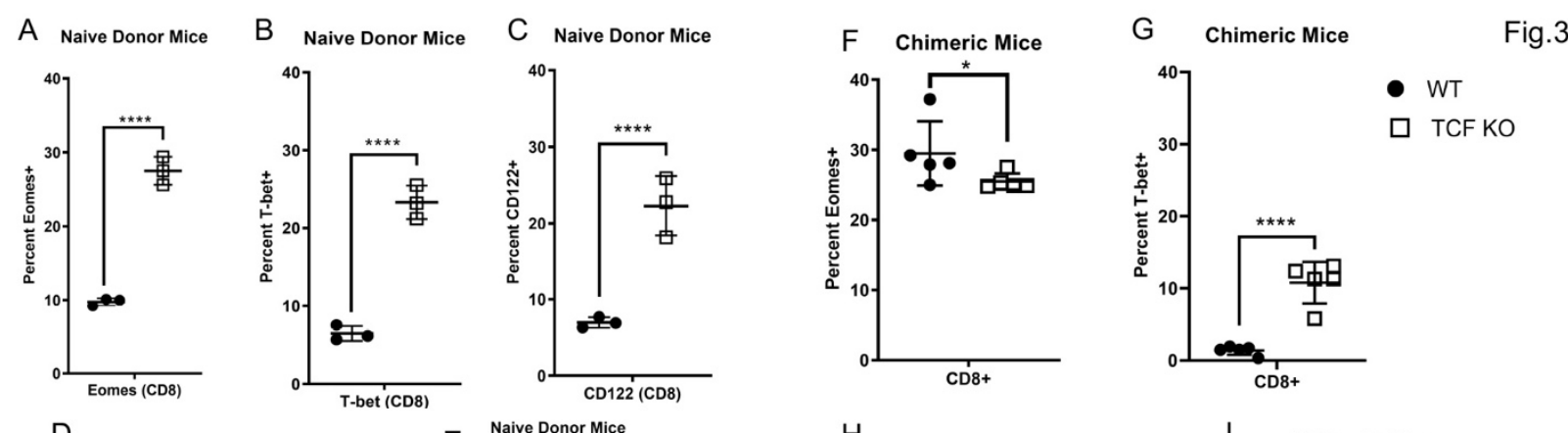

1078
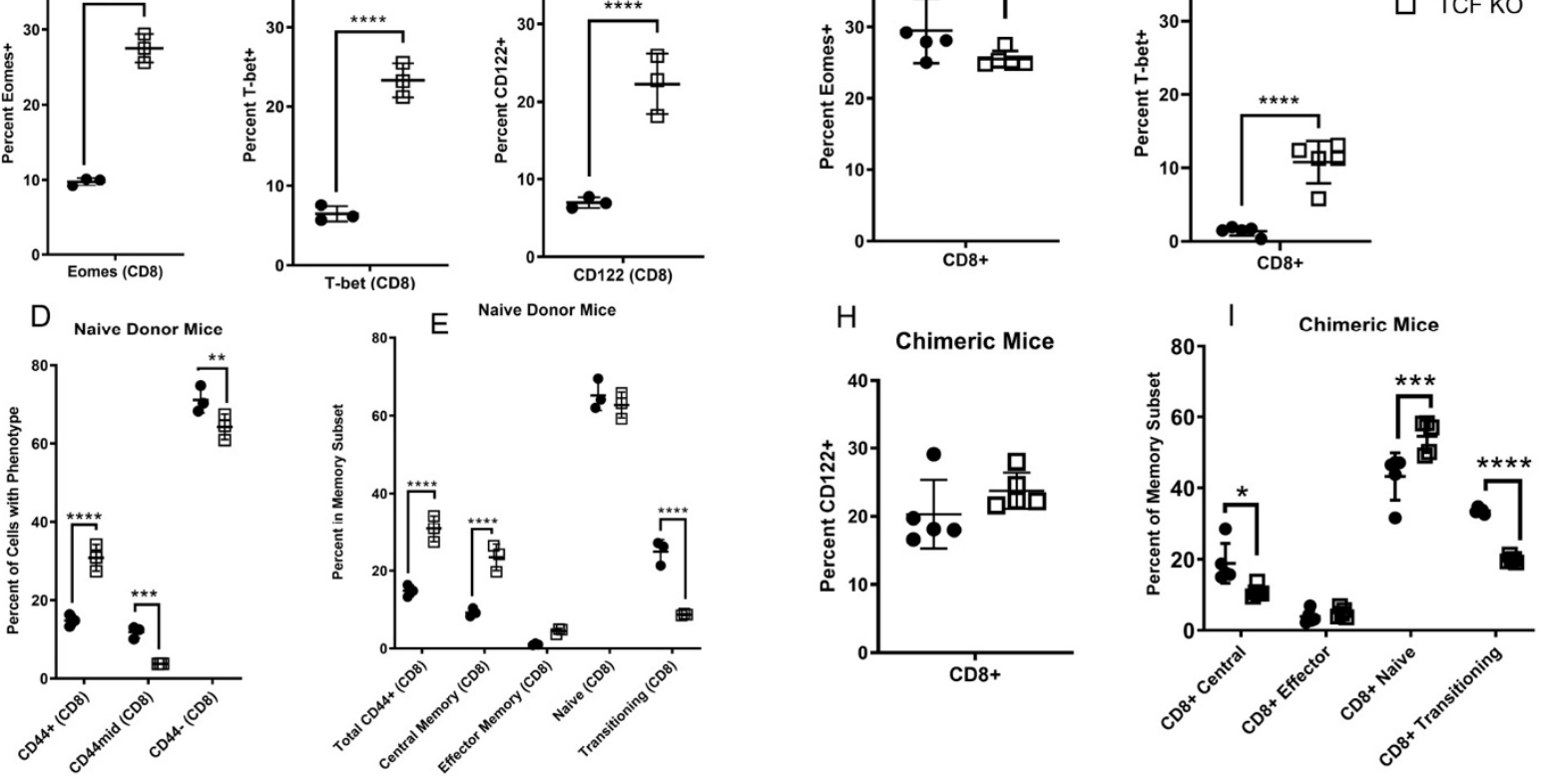

1079 Figure 3: Loss of TCF-1 drives changes to mature CD8 T cell phenotype which are

primarily cell-intrinsic. (A-E) Naive WT or TCF cKO donor mice were euthanized and 
1081 splenocytes were stained for flow cytometry phenotyping. (A) Percent of CD8 T cells expressing 1082 Eomes. (B) Percent of CD8 T cells expressing T-bet. (C) Percent of CD8 T cells expressing 1083 CD122. (D) Percent of CD8 T cells expressing CD44. (E) Percent of CD8 T cells expressing 1084 central memory, effector memory, transitioning/activating, or naive phenotypes. (F-I) Bone 1085 marrow chimeras were developed by lethally irradiating Thy1.1 mice and reconstituting with a 1086 1:4 (WT:TCF cKO) mixture of bone marrow cells. Blood was tested at 9 weeks to ensure 1087 reconstitution with both donor cell types, and splenocytes were used at 10 weeks for phenotyping 1088 by flow cytometry. (F) Percentage of chimeric CD8 T cells expressing Eomes. (G) Percentage of 1089 chimeric CD8 T cells expressing T-bet. (H) Percentage of chimeric CD8 T cells expressing 1090 CD122. (I) Percentage of chimeric CD8 T cells expressing central memory, effector memory, 1091 naive, or transitioning/activating phenotypes. All data are plotted as individual points with mean 1092 and SD, all were analyzed with one-way or two-way ANOVA, or Student's t-test (depending on 1093 groups). For all graphs, * means p-value $\leq 0.05, * * *$ means $\mathrm{p}$-value $\leq 0.001$, and $* * * *$ means $\mathrm{p}$ 1094 value $\leq 0.0001$. For A-E, N=3 per group with one representative experiment shown, for F-I, N=5 1095 with one experiment shown (done once). 

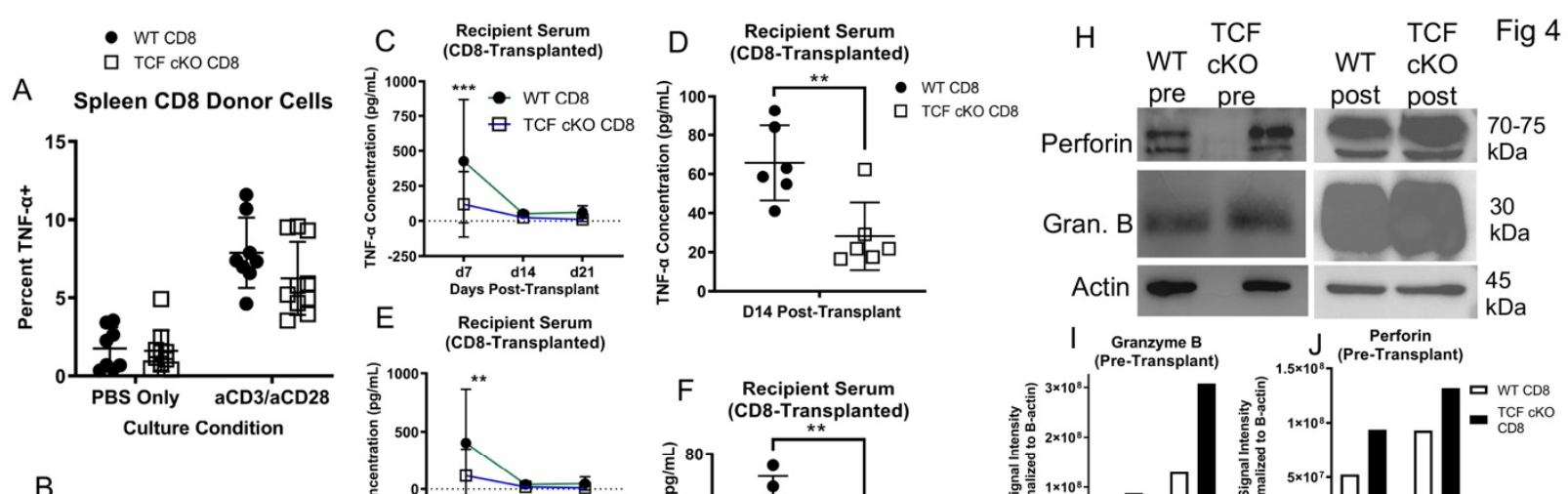

D14 Post-Transplant
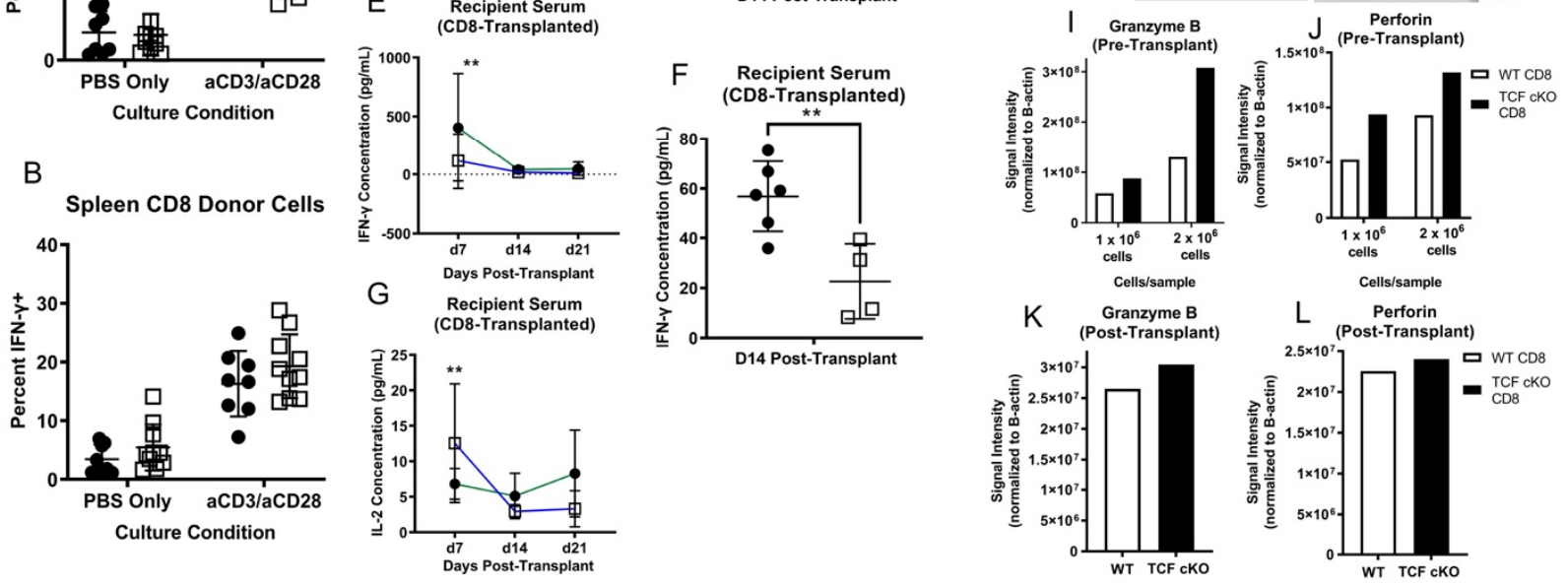

Figure 4: Loss of TCF-1 alters cytokine and cytotoxic mediator production by mature

alloactivated CD8 T cells. (A-B) Recipient mice were allotransplanted with $1.5 \times 10^{6}$ WT or

TCF cKO donor CD3 T cells and BALB/c BM, as before. Splenocytes were taken at d7 post-

1101 transplant, restimulated by 6hr culture with Golgi Plug and PBS (control) or anti-CD3/anti-CD28 (TCR restim.), then stained for $\mathrm{H} 2 \mathrm{~Kb}, \mathrm{CD} 3, \mathrm{CD} 4, \mathrm{CD} 8, \mathrm{TNF}-\alpha$, and IFN- $\gamma$. (A) TNF- $\alpha$ production and (B) IFN- $\gamma$ production by donor CD8 T cells, as measured by percent cytokinepositive donor cells. For A-B, individual points are plotted with mean and SD shown. (C-G) At day 7, day 14, and day 21 post-transplant, serum was obtained from cardiac blood of allotransplanted recipient mice and tested for various cytokines using a LEGENDplex ELISA assay. (C) Serum levels (pg/mL) of TNF- $\alpha$ for WT and TCF cKO-transplanted mice over time and (D) at day 14 only. (E) Serum levels (pg/mL) of IFN- $\gamma$ for WT and TCF cKO-transplanted 1109 mice over time and (F) at day 14 only. (G) Serum levels (pg/mL) of IL-2 over time for WT and 1110 TCF cKO-transplanted mice. For $\mathbf{C}, \mathbf{E}$, and $\mathbf{G}$, means with SD are plotted, for $\mathbf{D}$ and $\mathbf{F}$, 
1111 individual points with mean and SD are shown. All data in A-G were analyzed with Student's t-

1112 test or two-way ANOVA (depending on groups). ** means p-value $\leq 0.01$, and $* * *$ means $\mathrm{p}$ -

1113 value $\leq$ 0.001. (H-L) Pre-transplant and post-transplant donor CD8 T cells were isolated from

1114 donors or WT- or TCF cKO-transplanted recipient spleens and lysed for Western blot. (H) Blot

1115 photos showing bands for perforin, Granzyme B, and actin. Protein levels normalized to B-actin

1116 for (I) Granzyme B in pre-transplant cells, (J) perforin in pre-transplant cells, (K) Granzyme B

1117 in post-transplant cells, and (L) perforin in post-transplant cells. N=3-5 per group for A-B with

1118 two experiments shown, n=5-6 per group for $\mathbf{C}-\mathbf{G}$ with summary data $(\mathbf{C , E}, \mathbf{E})$ or points $(\mathbf{D , F})$

1119 shown from one representative experiment. H-L one well per condition as shown in blot, I-L one 1120 experiment plotted (done once).

1121
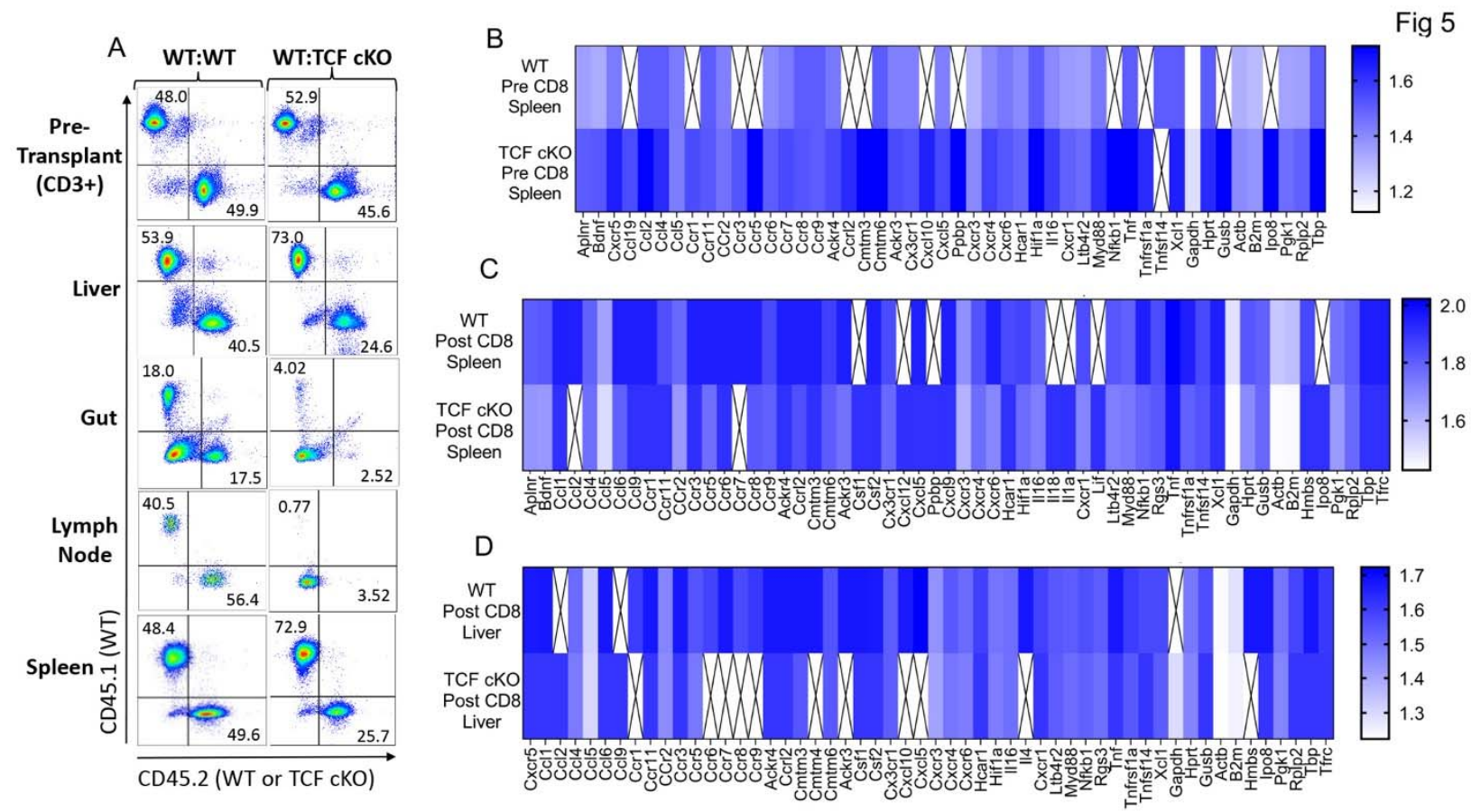

1122 Figure 5: TCF-1 controls chemokine and chemokine receptor expression of donor CD8 T

1123 cells during alloactivation. As before, recipient BALB/c mice were allotransplanted with

$1124 \mathrm{BALB} / \mathrm{c} \mathrm{BM}$ and donor CD3 T cells from WT and TCF cKO mice. Here, the $1 \mathrm{X} 10^{6}$ donor T 
1125 cells were a 1:1 mixture of WT (CD45.1):WT (CD45.2) or WT (CD45.1):TCF cKO (CD45.2)

1126 CD3 T cells. (A) Pre-transplant, a sample of donor cells was checked to ensure a 1:1 ratio of

1127 CD4 to CD8 T cells, and a 1:1 ratio of CD45.1:CD45.2 donor cells. At 7 days post-transplant,

1128 liver, gut, spleen, and lymph nodes were taken from euthanized recipient mice and processed to

1129 obtain lymphocytes. The lymphocytes were stained for $\mathrm{H} 2 \mathrm{~Kb}$ (donor cells), $\mathrm{H} 2 \mathrm{Kd}$ (recipient

1130 cells), CD3, CD4, CD8, CD45.1, and CD45.2. Pre- and post-transplant flow plots from one

1131 representative recipient are shown here. (B-D) BALB/c mice were allotransplanted with BM and

$11321 \times 10^{6}$ donor CD3 T cells from WT or TCF cKO mice (not mixed). Donor CD8 T cells were

1133 FACS-sorted from spleen of donors pre-transplant, and from spleen and liver of recipients at day

11347 post-transplant. Cells were sorted into Trizol, then RNA was extracted using chloroform and

1135 converted to cDNA for qPCR analysis. cDNA was run on premade mouse chemokine/chemokine

1136 receptor assay plates, and results are displayed as heatmaps. Scales are shown at right, with fold

1137 change per gene compared to an $18 \mathrm{~S}$ reference gene on each plate. White boxes with an "X"

1138 represent signals too low to detect or otherwise unreadable due to technical limitation/error. (B)

1139 Pre-transplant spleen donor CD8 T cells, (C) post-transplant spleen donor CD8 T cells, and (D)

1140 post-transplant liver donor CD8 T cells for WT versus TCF cKO donors. N=3 per group for A

1141 with one representative experiment shown, $\mathrm{N}=5$ mice into one sample per condition for B-D,

1142 summary data shown. 
- WT

口 TCF CKO

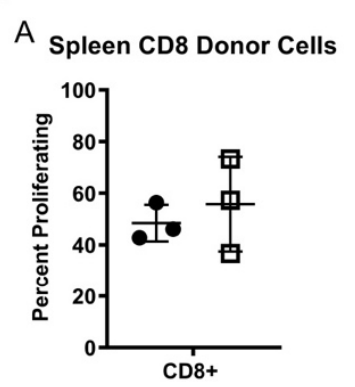

B Liver CD8 Donor Cells

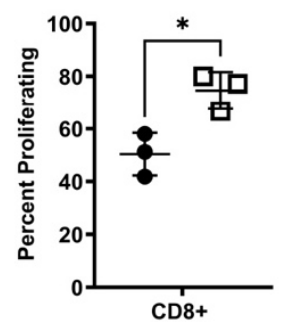

C Spleen CD8 Donor Cells

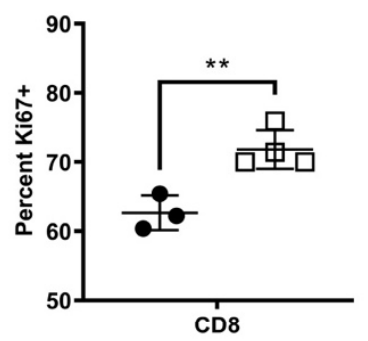

D Liver CD8 Donor Cells

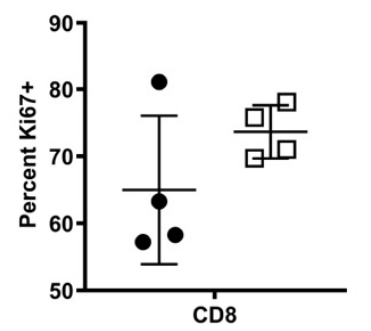

1143

1144

1145

1146

1147

1148

1149

1150

1151

1152

1153

1154

1155

1156

Figure 6: TCF-1 controls proliferation, apoptosis, and exhaustion of mature alloactivated
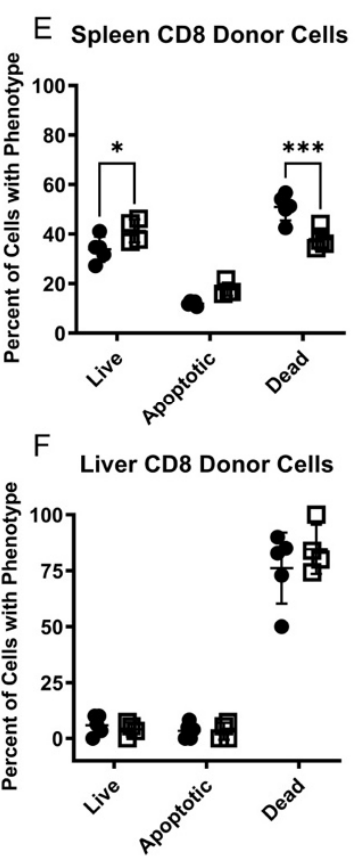

Fig.6

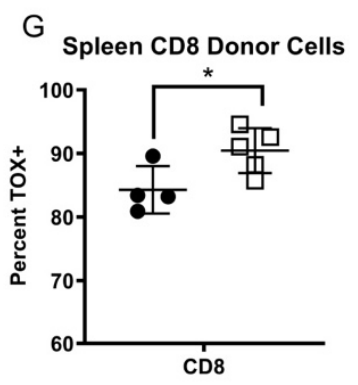

H Liver CD8 Donor Cells

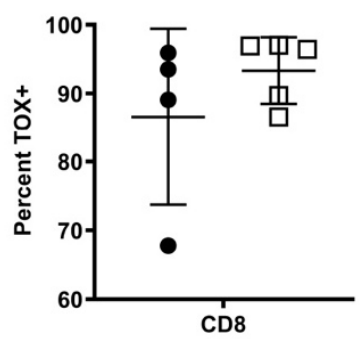

CD8 T cells. (A-B) BALB/c mice were allotransplanted as before, with BALB/c BM and WT or TCF cKO CD3 donor T cells. Recipient mice were injected i.p. with 25mg/kg EdU in PBS on day 5 and 6 post-transplant. On day 7, spleen and liver were removed from recipients, processed to isolate lymphocytes, and stained with an EdU click chemistry staining kit to detect EdU. Cells were also stained for CD3, CD4, CD8, and $\mathrm{H} 2 \mathrm{~Kb}$ to identify donor cells. (A) Percent of donor cells proliferating by EdU+ in spleen, and (B) in liver. (C-D) In a similar experiment, splenic and liver-derived lymphocytes from allotransplanted recipient mice were stained for CD3, CD4, CD8, $\mathrm{H} 2 \mathrm{~Kb}$, and Ki67 to identify proliferating/activated T cells. (C) Percent of donor CD8 cells proliferating by Ki67+ in spleen, and (D) in liver. (E-F) Recipient mice were allotransplanted as before with BM and WT or TCF cKO donor CD3 T cells. At day 7, spleen and liver were taken, processed to obtain lymphocytes, and stained for $\mathrm{CD} 3, \mathrm{CD} 4, \mathrm{CD} 8$, and $\mathrm{H} 2 \mathrm{~Kb}$, and with Annexin V-FITC, and LIVE/DEAD Near-IR. Cells were divided into three populations: live (Ann.V- 
1157 NIR-), dead (Ann.V+ NIR+), or apoptotic (Ann.V+NIR-). This was done for (E) spleen and (F)

1158 liver. (G-H) As before, recipient mice were allotransplanted, and at day 7, spleen and liver were

1159 removed and processed to obtain lymphocytes. Lymphocytes were stained for $\mathrm{H} 2 \mathrm{~Kb}, \mathrm{CD} 3, \mathrm{CD} 4$,

1160 CD8, and TOX to identify exhausted cells. This was done for (G) spleen and (H) liver. All data

1161 are shown as individual points with mean and SD, and were analyzed with Student's t-test or

1162 two-way ANOVA (depending on groups). * means p-value $\leq 0.05$, ** means p-value $\leq 0.01$, and

$1163 * * *$ means $\mathrm{p}$-value $\leq 0.001 . \mathrm{N}=3-5$ per group with one representative experiment shown.
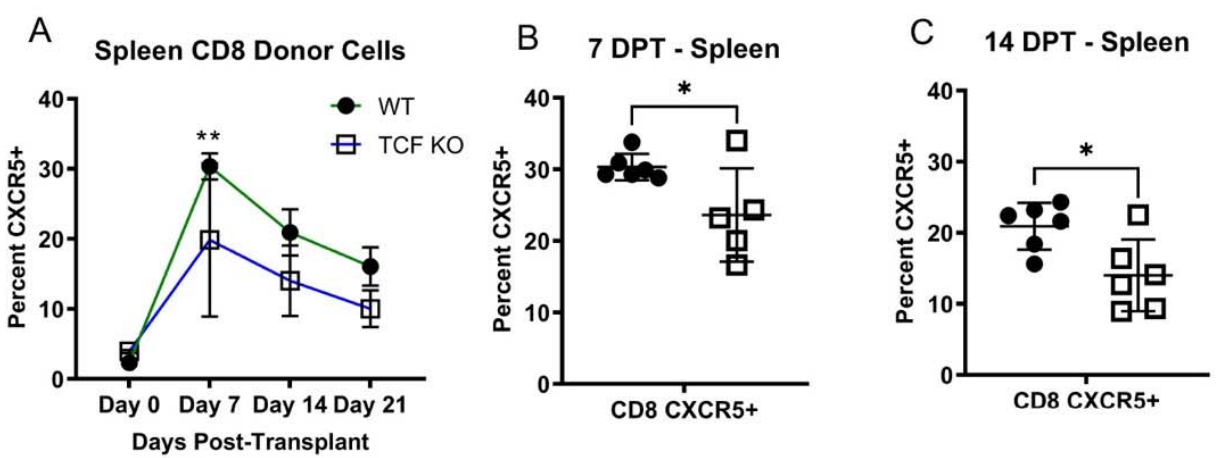

Fig.7
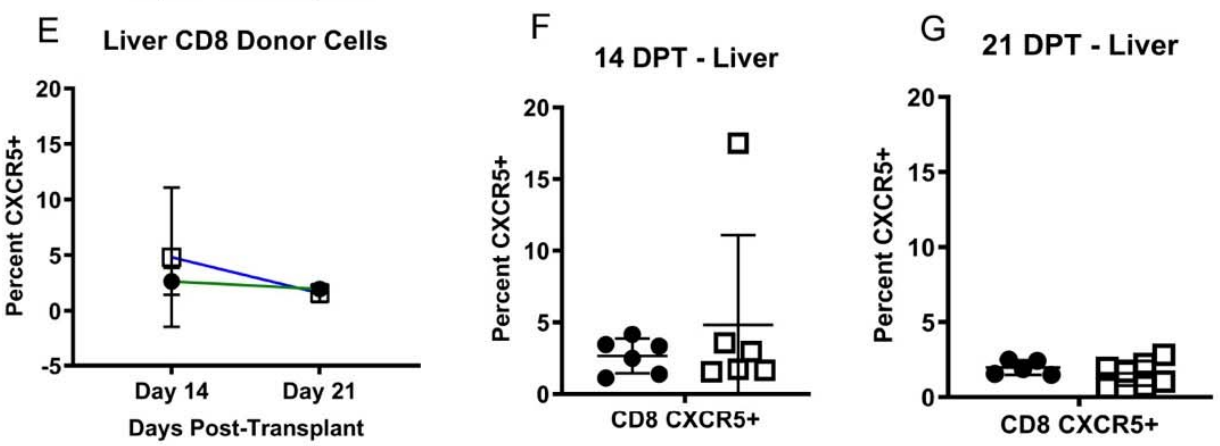

1164

Figure 7: Reduced CXCR5 expression in CD8 T cells of TCF-1 deficient mice may

1166 contribute to reduced GVHD severity. Recipient BALB/c mice were allotransplanted with

$1167 \mathrm{BALB} / \mathrm{c} \mathrm{BM}$ and WT or TCF cKO donor CD8 T cells. Prior to transplant, a sample of donor

1168 cells was stained for CXCR5. At day 7, day 14, and day 21 post-transplant, spleen and liver were

1169 taken from recipient mice, processed to obtain lymphocytes, and stained for CD3, CD4, CD8,

$1170 \mathrm{H} 2 \mathrm{~Kb}$, and CXCR5. (A) Expression of CXCR5 in spleen at day 0 and over time post-transplant. 
1171 (B-D) Expression of CXCR5 at each time point, (B) 7 days, (C) 14 days, and (D) 21 days post-

1172 transplant in the spleen. (E) Expression of CXCR5 in liver-derived donor cells over time post-

1173 transplant. (F-G) Expression of CXCR5 at (F) day 14 and (G) day 21 post-transplant in the liver.

1174 N=5-6 per group and condition, with summary data $(\mathbf{A}, \mathbf{E})$ or individual points $(\mathbf{B}-\mathbf{D}, \mathbf{F}-\mathbf{G})$ shown

1175 for one experiment (done once).
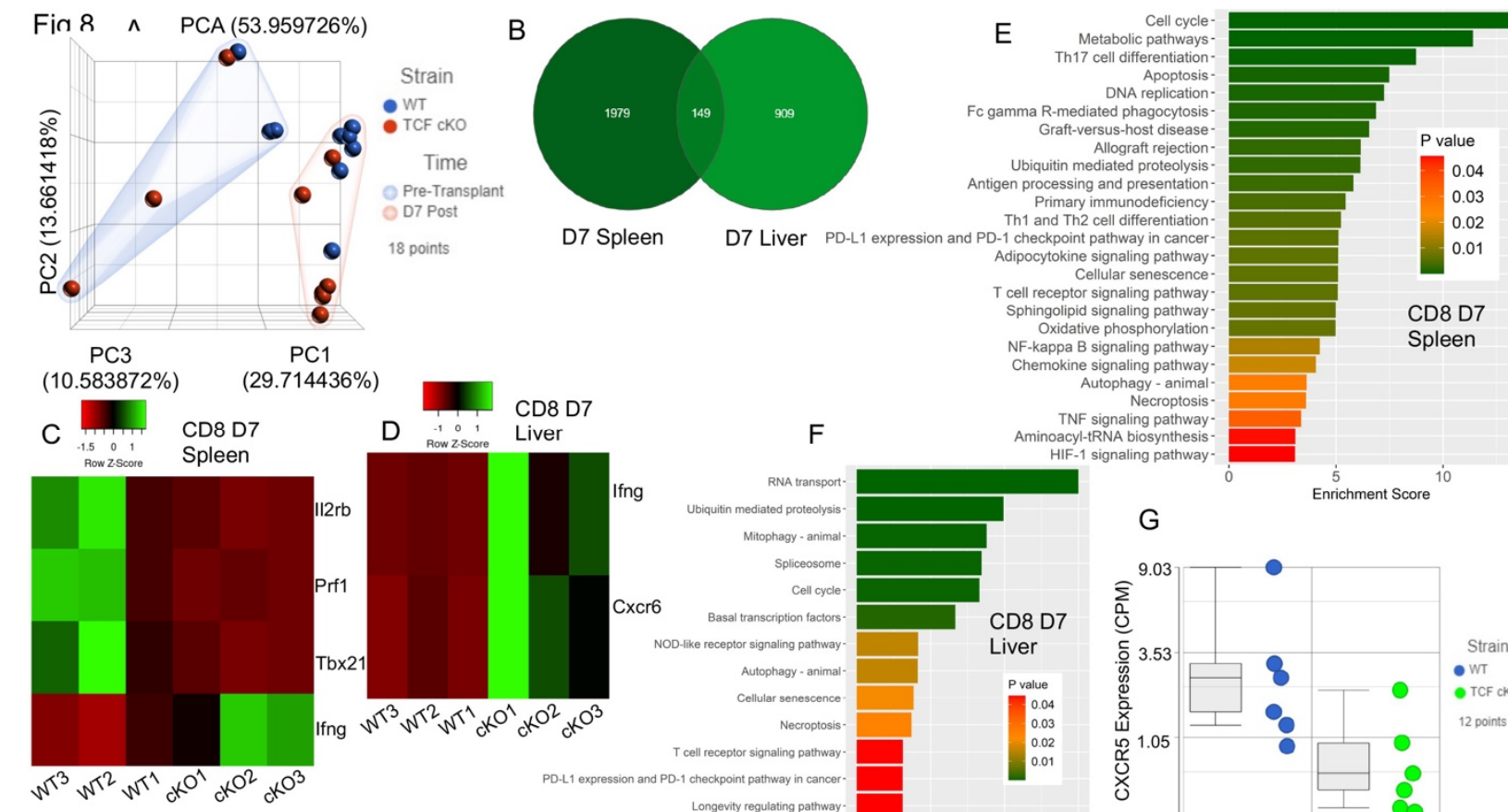

1176

Figure 8: TCF-1 contributes to control of cell survival and immune signaling pathways in

1178 CD8 donor T cells. To perform RNA sequencing, recipient BALB/c mice were allotransplanted

1179 with BALB/c BM and WT or TCF cKO CD3 donor T cells. Pre-transplant CD8+ donor cells

1180 were FACS-sorted to obtain a sample for sequencing. At day 7 post-transplant, donor CD8 T

1181 cells were FACS-sorted back from spleen and liver of recipient mice. Cells were sorted into

1182 Trizol, then RNA was extracted and paired-end sequencing was performed. (A) PCA analysis

1183 showing clustering of donor CD8 T cells by strain and by timepoint (pre- and post-transplant).

1184 Points are colored by strain and grouped by timepoint. (B) Venn diagram showing number of 
1185 differentially expressed genes found in post-transplant spleen and liver for WT versus TCF cKO

1186 CD8 donor T cells. FDR $\leq 0.05$ and fold change $\geq 2$ or $\leq-2$ were used as criteria for selection.

1187 (C-D) Heatmap showing select significantly changed genes in donor CD8 T cells from (C) post-

1188 transplant spleen, or (D) post-transplant liver. (E-F) Pathway enrichment analysis, with selected

1189 enriched pathways shown for (E) post-transplant liver and (F) post-transplant spleen. All

1190 pathways shown have $\mathrm{p}$-value $\geq 0.05$, and fill color indicates p-value. (G) Expression level of

1191 CXCR5 in counts per million reads for WT versus TCF cKO donor CD8 T cells, post-transplant,

1192 in spleen and liver. Individual samples are shown, with boxplot indicating summary statistics.

1193 Log scale is used on y-axis. N=3-5 samples per group and condition, one experiment shown

1194 (done once).

1195 


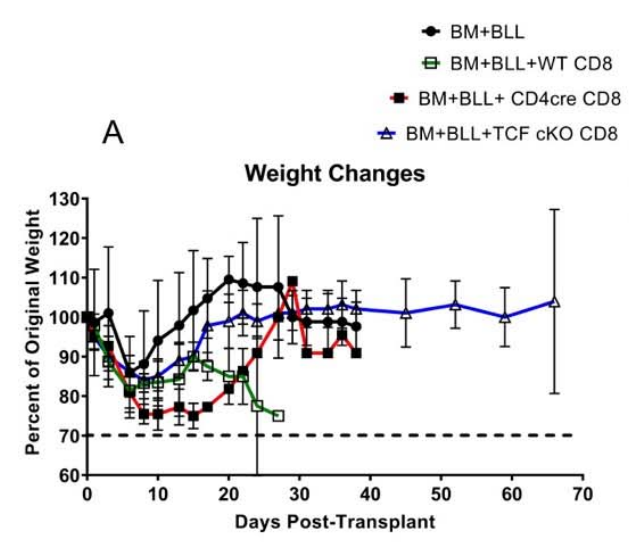

B

B

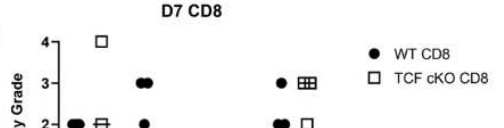

C

Supp.fig.1

1198

Supplemental Figure 1: GVHD symptoms and organ damage caused by donor CD8 T cells

are reduced by loss of TCF-1, related to Figure 1. Recipient BALB/c mice were

allotransplanted with BALB/c BM and WT, CD4cre, or TCF cKO CD8 T cells as described in

Fig. 1. (A) Weight changes for each group of mice over the experiment, mean and SD are plotted. (B) GVHD histology score for organ sections (liver, spleen, skin, and small intestine) were scored by a pathologist (A.M.) blinded to study conditions and slide identity. Individual and day 21 are shown. $\mathrm{N}=3-5$ per group with one representative experiment shown in $\mathrm{A}$, 


\section{Supp.fig.2}

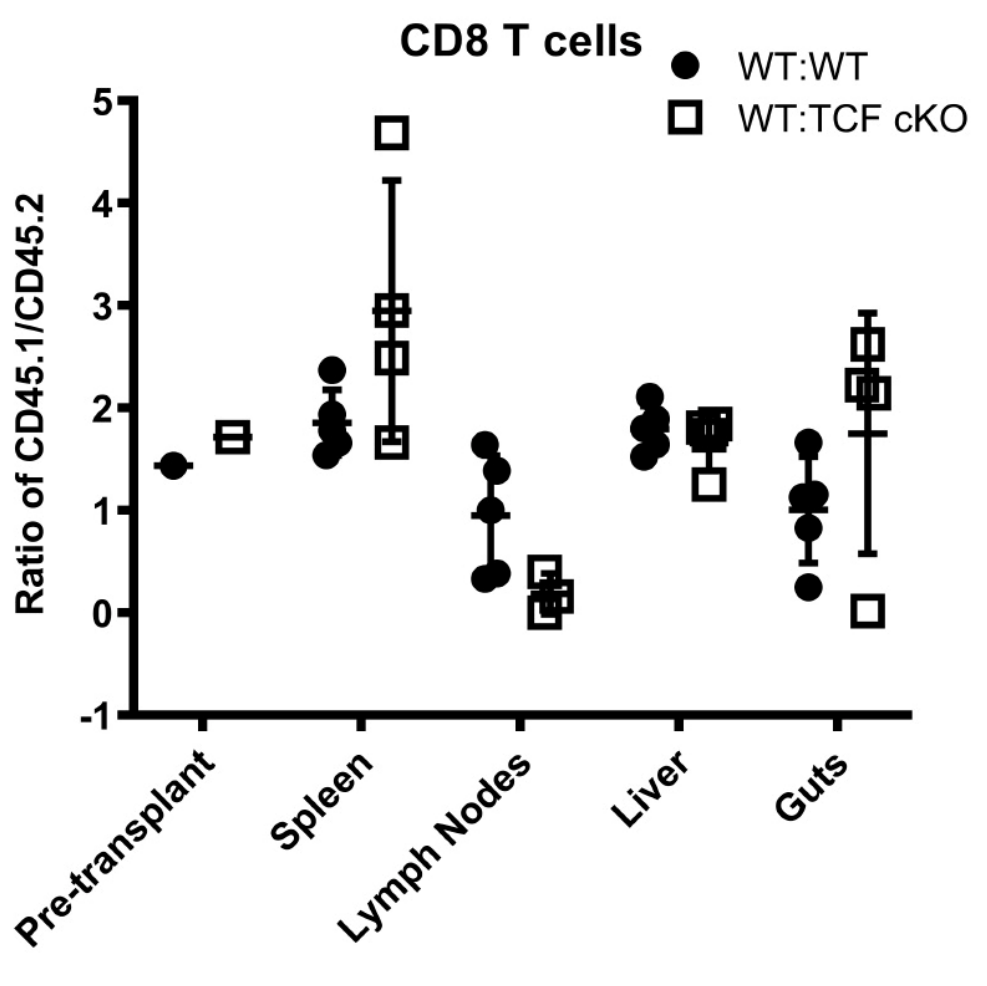
cells, related to Figure 5. Recipient BALB/c mice were allotransplanted with BALB/c BM and

1212 cells (total $1 \times 10^{6}$ cells). Prior to transplant and at day 7 post-transplant, the ratio of CD45.1 to

1213 CD45.2 donor cells was assessed by flow cytometry. Pre-transplant samples were taken from

1214 cells to be transplanted, and day 7 samples were obtained from recipient spleen, lymph nodes,

1215 liver, and small intestine. Individual points are plotted, with mean and SD shown. Data were

1216 analyzed with two-way ANOVA. $\mathrm{N}=4-5$ per group ( $\mathrm{n}=1$ for pre-transplant), one representative 1217 experiment shown. 

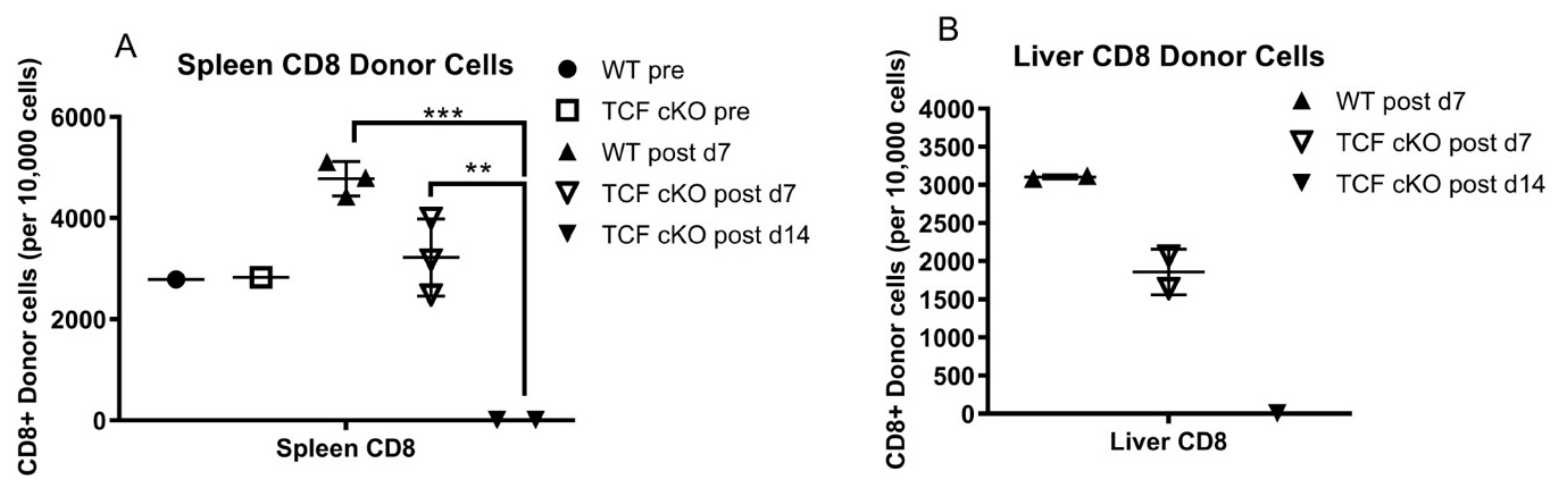

1221 donor T cells. Donor CD8 T cells were FACS-sorted from pre-transplant donors or from

1222 recipient spleen and liver 7 days post-transplant. TCF cKO donor cells only were also sorted at 1223 day 14 from spleen and liver. Number of CD8+ donor cells obtained by sorting per 10,000 sorted

1224 cells is shown in (A) spleen and (B) liver. Individual points are shown with mean and SD, data

1225 were analyzed by two-way ANOVA. ** means p-value $\leq 0.01$, and $* * *$ means $p$-value $\leq 0.001$.

$1226 \mathrm{~N}=2-3$ per group ( $\mathrm{n}=1$ for pre-transplant), one representative experiment shown. 


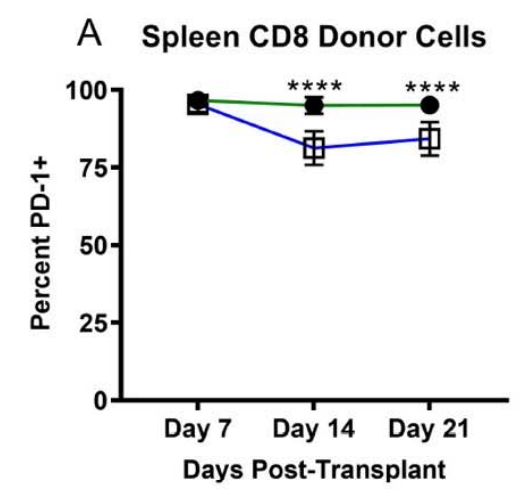

B

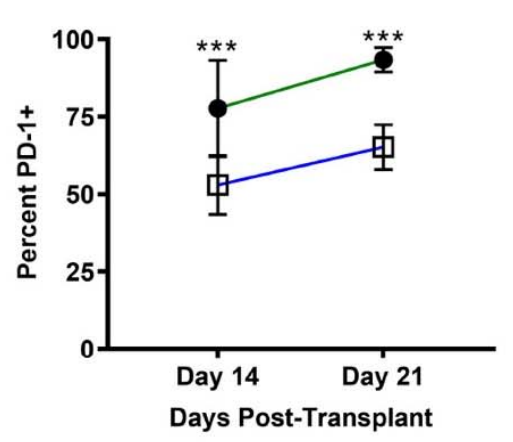

C Spleen CD8 Donor Cells

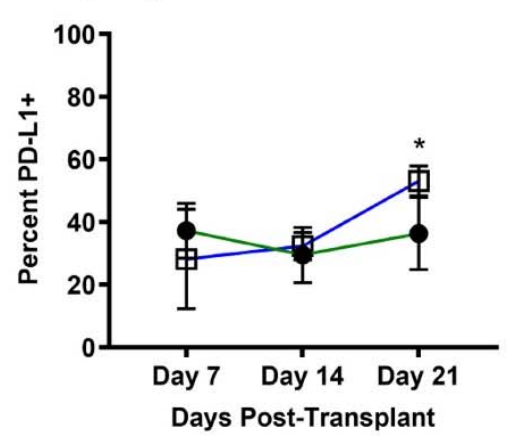

D Liver CD8 Donor Cells

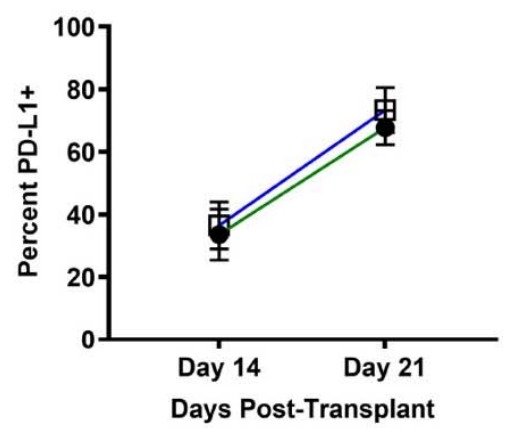

Supp fig 4

E Spleen CD8 Donor Cells

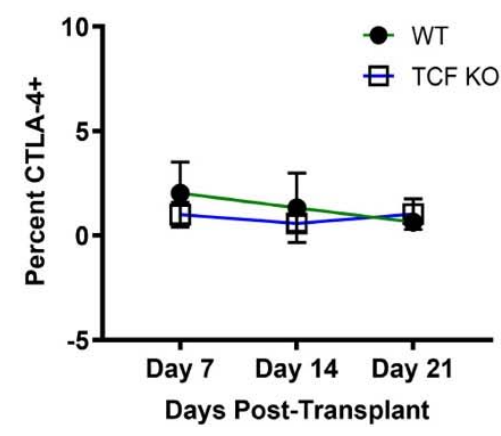

F Liver CD8 Donor Cells

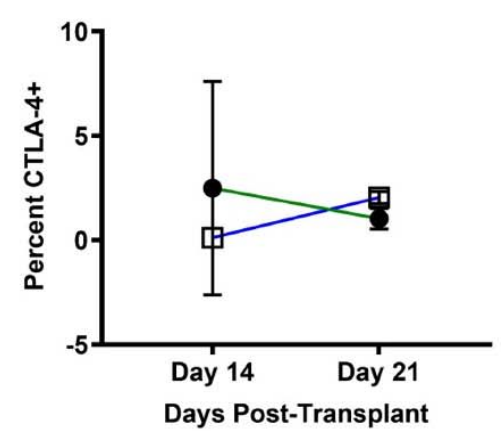

Supplemental Figure 4: Loss of TCF-1 changes the suppressive profile of CD8 donor T

1229

1230

1231

1232

1233

1234

1235

1236

1237

cells, related to Figure 6. Recipient BALB/c mice were allotransplanted with BALB/c BM and

WT or TCF cKO donor CD8 T cells. At day 7, day 14, and day 21 post-transplant, spleen and liver were taken from recipients and processed to obtain lymphocytes. Lymphocyes were stained for CD3, CD4, CD8, H2Kb, PD-1, PD-L1, and CTLA-4. (A) Expression of PD-1 on CD8+ donor cells in spleen over time. (B) Expression of PD-1 on CD8+ donor cells in liver over time. (C) Expression of PD-L1 on CD8+ donor cells in spleen over time. (D) Expression of PD-L1 on CD8+ donor cells in liver over time. (E) Expression of CTLA-4 on CD8+ donor cells in spleen over time. (F) Expression of CTLA-4 on CD8+ donor cells in liver over time. Mean and SD are shown, data were analyzed with two-way ANOVA, * means p-value $\leq 0.05, * * *$ means p-value 
$1238 \leq 0.001$, and $* * * *$ means $\mathrm{p}$-value $\leq 0.0001 . \mathrm{N}=3-4$ per group and timepoint, summary data from

1239 one experiment shown (done once).

1240
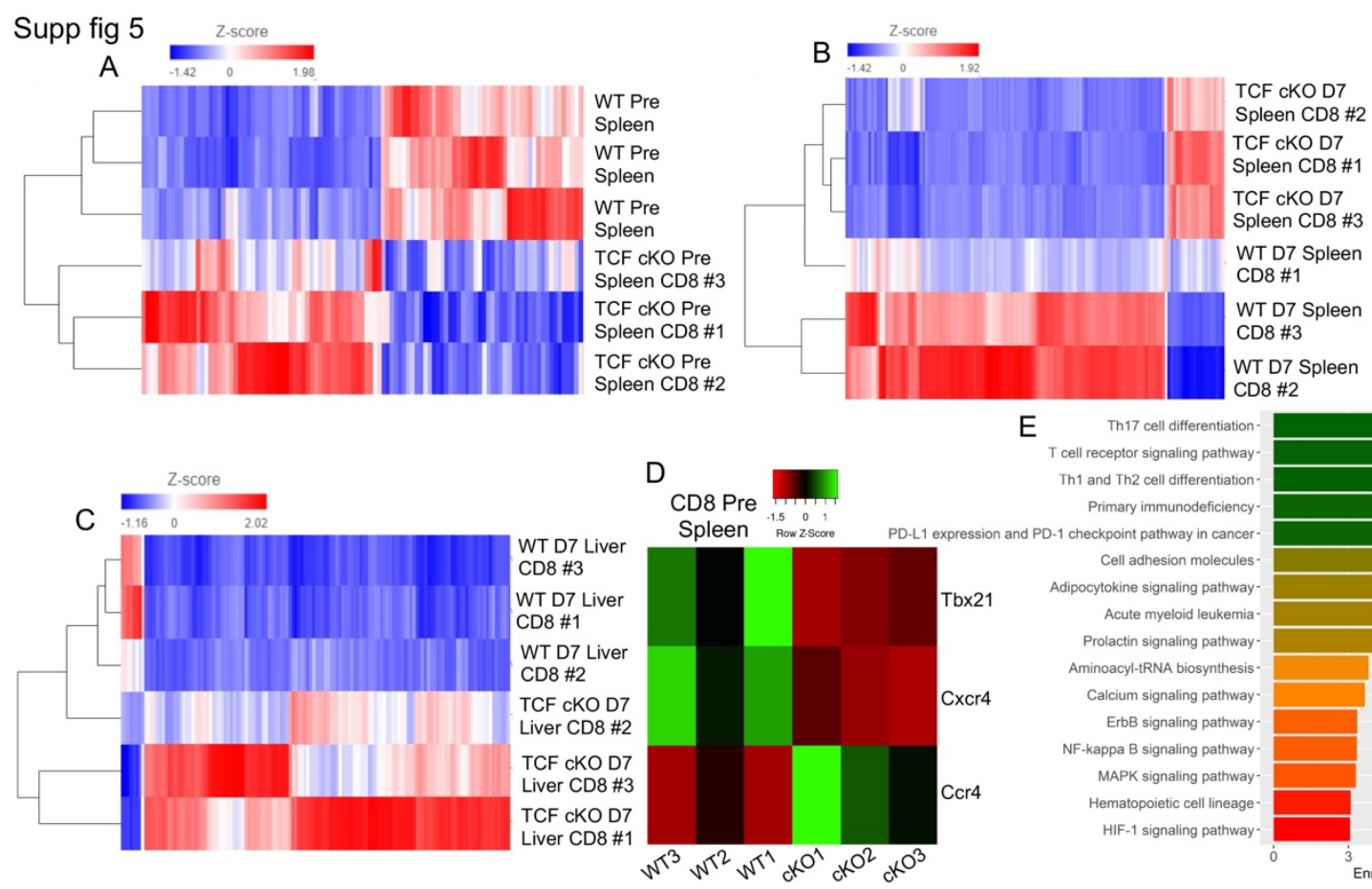

E Th17 cell differentiation

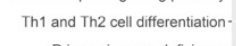

Primary immunodeficiency.

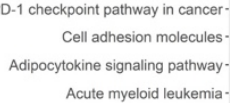

Acute myeloid leukemia

Prolactin signaling pathwayAminoacyl-tRNA biosynthesis -

Calcium signaling pathway NF-kappa B signaling pathway-

MAPK signaling pathway-

Hematopoietic cell lineage

HIF-1 signaling pathway -

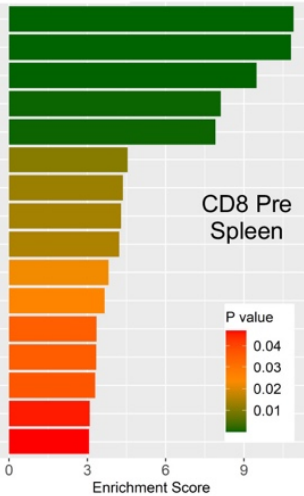

1241 Supplemental Figure 5: Donor CD8 T cell samples cluster by strain, and TCF-1 alters pre-

1242 transplant gene expression, related to Figure 8. As for Fig. 8, recipient mice were

1243 allotransplanted with BM and WT or TCF cKO donor CD3 T cells. Pre- and day 7 post-

1244 transplant donor cells were FACS-sorted from donor spleen or recipient spleen/liver,

1245 respectively. RNA sequencing was performed on donor cells as in Fig. 8. (A-C) Hierarchical

1246 clustering was performed for (A) pre-transplant spleen, (B) post-transplant spleen, and (C) post-

1247 transplant liver samples. (D) Heatmap showing select significantly changed genes in donor CD8

1248 T cells from pre-transplant spleen. (E) Pathway analysis was performed on pre-transplant CD8

1249 donor T cell samples from spleen. Differentially expressed genes for D-E were identified by p- 
bioRxiv preprint doi: https://doi.org/10.1101/2021.03.22.436494; this version posted March 23, 2021. The copyright holder for this preprint (which was not certified by peer review) is the author/funder. All rights reserved. No reuse allowed without permission.

1250 value $\leq 0.05$ (rather than FDR $\leq 0.05$ ) and fold change $\geq 2$ or $\leq-2$. N=3 per group and condition, 1251 one experiment shown (done once).

1252 
CD8+ Donor T Cells

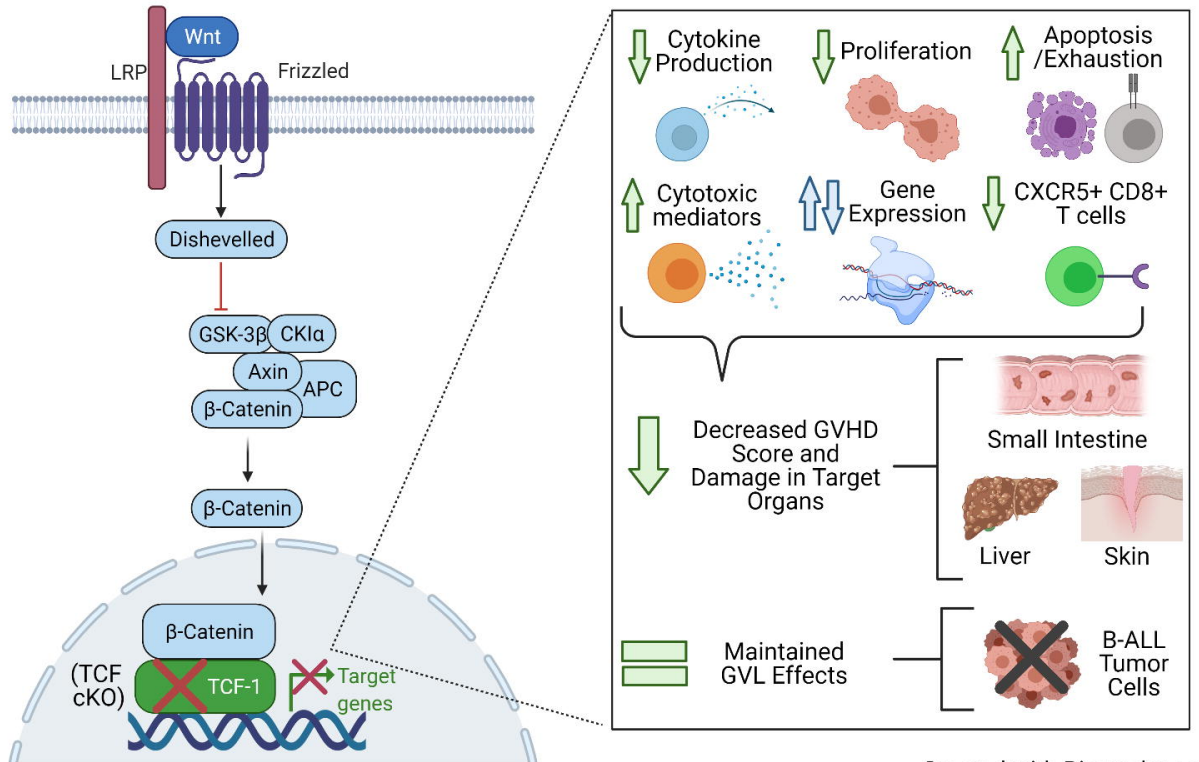

